\title{
A priori error estimates for a time-dependent boundary element method for the acoustic wave equation in a half-space
}

\author{
Heiko Gimperlein, Zouhair Nezhi and Ernst P. Stephan
}

\begin{abstract}
We investigate a time-domain Galerkin boundary element method for the wave equation outside a Lipschitz obstacle in an absorbing half-space, with application to the sound radiation of tyres. A priori estimates are presented for both closed surfaces and screens, and we discuss the relevant properties of anisotropic Sobolev spaces and the boundary integral operators between them.
\end{abstract}

\section{Introduction}

Motivated by the sound radiation of tyres on a street, this article provides the analytical background to analyze a time-domain boundary element method for the direct scattering problem for the wave equation outside an obstacle in an absorbing half-space.

Let $d \geq 2$ and $\Omega^{i} \subset \mathbb{R}_{+}^{d}$ be a bounded Lipschitz domain such that the exterior domain $\Omega^{e}=\mathbb{R}_{+}^{d} \backslash \overline{\Omega^{i}}$ is Lipschitz and connected. The reader may wish to think of $\Omega^{i}$ as a solid tyre, either in contact with the street (on $\partial \Omega^{i} \cap \partial \mathbb{R}_{+}^{d}$ ) or elevated above it $\left(\partial \Omega^{i} \cap \partial \mathbb{R}_{+}^{d}=\emptyset\right)$. The boundary of $\Omega^{e}$ decomposes into the boundary $\Gamma=\partial \Omega^{e} \cap \partial \Omega^{i}$ of the obstacle and the boundary $\Gamma_{\infty}=\partial \Omega^{e} \cap \partial \mathbb{R}_{+}^{d}$ of the half-space. In general, $\Gamma$ is a Lipschitz manifold with boundary, and we emphasize the case $d=3$.

We aim to find a weak solution to an acoustic initial boundary problem for the wave equation in $\Omega^{e}$ :

$$
\begin{aligned}
\frac{\partial^{2} u}{\partial t^{2}}-\Delta u & =0 & & \text { in } \mathbb{R}^{+} \times \Omega^{e} \\
u(0, x)=\frac{\partial u}{\partial t}(0, x) & =0 & & \text { in } \Omega^{e} \\
\frac{\partial u}{\partial n}-\alpha \frac{\partial u}{\partial t} & =g & & \text { on } \mathbb{R}^{+} \times \Gamma \\
\frac{\partial u}{\partial n}-\alpha_{\infty} \frac{\partial u}{\partial t} & =0 & & \text { on } \mathbb{R}^{+} \times \Gamma_{\infty} .
\end{aligned}
$$


Here $n$ denotes the inward unit normal vector to $\partial \Omega^{e}, g$ lies in a suitable Sobolev space, $\alpha \in L^{\infty}(\Gamma)$ and $\alpha_{\infty} \in \mathbb{C}$. We also consider the simpler Dirichlet problem on $\Gamma$, for which instead of the absorbing boundary condition, $\left.u\right|_{\mathbb{R}^{+} \times \Gamma}$ is given.

This article reduces the acoustic and Dirichlet boundary problems to timedependent integral equations on $\mathbb{R}^{+} \times \Gamma$ and studies a Galerkin time-domain boundary element method for their approximation. Time-dependent Galerkin boundary element methods for wave problems were introduced by Bamberger and Ha-Duong [1. Some relevant works on the numerical implementation of the resulting marching-in-on-time scheme include the Ph.D. thesis of Terrasse [2] and [3, with fast methods developed in the engineering literature [4]. Alternative ansatz functions in time have been explored in [5, 6]. A detailed exposition of the mathematical background of time-domain integral equations and their discretizations is available in the lecture notes by Sayas [7].

In the special case of the half-space, our work is motivated by the recent explicit formulas for the fundamental solutions obtained by Ochmann [8], which include acoustic boundary conditions on the surface of the street.

Section 2 introduces space-time anisotropic Sobolev spaces of supported resp. extendable distributions on $\mathbb{R}^{+} \times \Gamma$. Their approximation theory and interpolation operators are the subject of Section 3. Subsequent sections follow the approach of Bamberger and Ha Duong [1, 9], see also [10], to analyze the coercivity and boundedness properties of time-dependent layer potentials adapted to the acoustic boundary conditions on $\Gamma_{\infty}$. As conclusion, we deduce a priori error estimates for the Galerkin solutions.

The results in this article provide a basic theoretical background for further theoretical and computational analysis. Based on the set-up presented here, future work will address a posteriori error estimates and adaptive procedures [11] as well as numerical studies of engineering benchmarks [12].

We acknowledge support within the project "LeiStra3" by the German Bundesministerium für Wirtschaft und Energie as well as the Bundesanstalt für Straßenwesen. H.G. thanks the Danish Science Foundation (FNU) for partial support through research grant 10-082866.

Notation: To simplify notation, we will write $f \lesssim g$, if there exists a constant $C>0$ independent of the arguments of the functions $f$ and $g$ such that $f \leq C g$. We will write $f \lesssim_{\sigma} g$, if $C$ may depend on $\sigma$.

\section{Space-time anisotropic Sobolev spaces}

Space-time anisotropic Sobolev spaces on the boundary $\Gamma$ provide a convenient setting to study the mapping properties of the layer potentials [9, 10. We define these Sobolev spaces on a general $C^{k-1,1}$ closed, orientable manifold $\mathcal{M}$ with boundary, in particular obtain Sobolev spaces on $\mathcal{M}=\Gamma$ and on $\mathcal{M}=\Omega$. 
The isotropic case is well-known from elliptic problems, see [13], and [14] when $\partial \mathcal{M} \neq \emptyset$.

If $\partial \mathcal{M} \neq \emptyset$, first extend $\mathcal{M}$ to a $C^{k-1,1}$, closed, orientable manifold $\widetilde{\mathcal{M}}$. For example, if $\mathcal{M}=\partial \Omega \cap \mathbb{R}_{+}^{d}$ and $k=1$, we may take $\widetilde{\mathcal{M}}$ to be union of $\partial \Omega \cap \overline{\mathbb{R}_{+}^{d}}$ and its image under reflection at $\partial \mathbb{R}_{+}^{d}$.

On $\mathcal{M}$, the usual Sobolev spaces of supported distributions may now be considered for $r \in \mathbb{R}$ :

$$
\widetilde{H}^{r}(\mathcal{M})=\left\{u \in H^{r}(\widetilde{\mathcal{M}}): \operatorname{supp} u \subset \overline{\mathcal{M}}\right\} .
$$

$H^{r}(\mathcal{M})$ is defined as the quotient space $H^{r}(\widetilde{\mathcal{M}}) / \widetilde{H}^{r}(\widetilde{\mathcal{M}} \backslash \overline{\mathcal{M}})$.

To define a family of Sobolev norms, introduce a partition of unity subordinate to the covering of $\widetilde{\mathcal{M}}$ by open sets $B_{i}$. For a partition of unity $\alpha_{i}$ and diffeomorphisms $\varphi_{i}$ mapping each $B_{i}$ into the unit cube $Q \subset \mathbb{R}^{n}$, a family of equivalent Sobolev norms is induced from $\mathbb{R}^{n}$ :

$$
\|u\|_{r, \omega, \widetilde{\mathcal{M}}}=\left(\sum_{i=1}^{p} \int_{\mathbb{R}^{n}}\left(|\omega|^{2}+|\xi|^{2}\right)^{r}\left|\mathcal{F}\left\{\left(\alpha_{i} u\right) \circ \varphi_{i}^{-1}\right\}(\xi)\right|^{2} d \xi\right)^{\frac{1}{2}}
$$

Here $\omega \in \mathbb{C} \backslash\{0\}$ and $\mathcal{F}$ denotes the Fourier transform. This norm on $H^{r}(\widetilde{\mathcal{M}})$ induces a norm on $H^{r}(\mathcal{M})$ as $\|u\|_{r, \omega, \mathcal{M}}=\inf _{v \in \widetilde{H}^{r}(\widetilde{\mathcal{M}} \backslash \overline{\mathcal{M}})}\|u+v\|_{r, \omega, \widetilde{\mathcal{M}}}$.

The weighted norm on $\widetilde{H}^{r}(\mathcal{M})$ is defined as $\|u\|_{r, \omega, \mathcal{M}, *}=\left\|e_{+} u\right\|_{r, \omega, \widetilde{\mathcal{M}}}$, where $e_{+}$extends the distribution $u$ by 0 from $\mathcal{M}$ to $\widetilde{\mathcal{M}}$. It is stronger than $\|u\|_{r, \omega, \mathcal{M}}$ whenever $r \in \frac{1}{2}+\mathbb{Z}$.

For $|r| \leq k$ the thus defined Sobolev spaces are independent of the choice of $\alpha_{i}$ and $\varphi_{i}$.

Using these norms, the trace operator from $H^{1}(\Omega)$ to $H^{\frac{1}{2}}(\Gamma)$ is continuous in the $\omega$-dependent norms. As shown in [9], for $\sigma>0$ its operator norm is uniformly bounded in the half-plane $\{\omega \in \mathbb{C}: \operatorname{Im} \omega>\sigma\}$ by a function of $\sigma$ alone. It admits a right inverse from $H^{\frac{1}{2}}(\Gamma)$ and $\widetilde{H}^{\frac{1}{2}}(\Gamma)$ to $H^{1}(\Omega)$ whose norm is similarly bounded in terms of $\sigma$.

Let $E$ be a Hilbert space. We define

$$
L T(\sigma, E)=\left\{f \in \mathcal{D}_{+}^{\prime}(E) ; e^{-\sigma t} f \in \mathcal{S}_{+}^{\prime}(E)\right\},
$$

where $\mathcal{D}_{+}^{\prime}(E)$ resp. $\mathcal{S}_{+}^{\prime}(E)$ denote the sets of distributions resp. tempered distributions on $\mathbb{R}$ with values in $E$ and support in $[0, \infty)$. As $L T(\sigma, E) \subset L T\left(\sigma^{\prime}, E\right)$ if $\sigma<\sigma^{\prime}$, we may define $\sigma(f)=\inf \{\sigma: f \in L T(\sigma, E)\}$.

The set of Laplace transformable distributions with values in $E$ is denoted by

$$
L T(E)=\cup_{\sigma \in \mathbb{R}} L T(\sigma, E)
$$


For $f \in L T(E)$, its Fourier-Laplace transform $\hat{f}(\omega)=\mathcal{F} f(\omega)$ is defined in the complex half-plane $\{\omega \in \mathbb{C}: \operatorname{Im} \omega>\sigma(f)\}$.

We recall the well-known Parseval identity in this setting:

Lemma 2.1. For $f, g \in L_{l o c}^{1}(\mathbb{R}, E) \cap L T(E)$ and $\sigma>\max (\sigma(f), \sigma(g))$ there holds

$$
\frac{1}{2 \pi} \int_{\mathbb{R}+i \sigma}(\hat{f}(\omega), \hat{g}(\omega))_{E} d \omega=\int_{\mathbb{R}} e^{-2 \sigma t}(f(t), g(t))_{E} d t
$$

Space-time anisotropic Sobolev spaces on $\mathcal{M}$ are now defined as follows:

Definition 2.2. For $s, r \in \mathbb{R}$ define

$$
\begin{array}{r}
H_{\sigma}^{s}\left(\mathbb{R}^{+}, H^{r}(\mathcal{M})\right)=\left\{u \in L T\left(H^{r}(\mathcal{M})\right):\|u\|_{s, r, \mathcal{M}}<\infty\right\} \\
H_{\sigma}^{s}\left(\mathbb{R}^{+}, \widetilde{H}^{r}(\mathcal{M})\right)=\left\{u \in L T\left(\widetilde{H}^{r}(\mathcal{M})\right):\|u\|_{s, r, \mathcal{M}, *}<\infty\right\},
\end{array}
$$

where

$$
\begin{aligned}
\|u\|_{s, r, \mathcal{M}} & =\left(\int_{-\infty+i \sigma}^{+\infty+i \sigma}|\omega|^{2 s}\|\hat{u}(\omega)\|_{r, \omega, \mathcal{M}}^{2} d \omega\right)^{\frac{1}{2}}, \\
\|u\|_{s, r, \mathcal{M}, *} & =\left(\int_{-\infty+i \sigma}^{+\infty+i \sigma}|\omega|^{2 s}\|\hat{u}(\omega)\|_{r, \omega, \mathcal{M}, *}^{2} d \omega\right)^{\frac{1}{2}} .
\end{aligned}
$$

As above, the spaces are invariantly defined whenever $|r| \leq k$.

\section{Discretisation}

For simplicity of notation, in this section we restrict ourselves to the two- and three-dimensional cases, $d=2$ or 3 . If $\Gamma$ is not polygonal we approximate it by a piecewise polygonal curve resp. surface and write $\Gamma$ again for the approximation. For simplicity, when $d=3$ we will use here a surface composed of $N$ triangular facets $\Gamma_{i}$ such that $\Gamma=\cup_{i=1}^{N} \Gamma_{i}$. When $d=2$, we assume $\Gamma=\cup_{i=1}^{N} \Gamma_{i}$ is composed of line segments $\Gamma_{i}$. In each case, the elements $\Gamma_{i}$ are closed with $\operatorname{int}\left(\Gamma_{i}\right) \neq \varnothing$, and for distinct $\Gamma_{i}, \Gamma_{j} \subset \Gamma$ the intersection $\operatorname{int}\left(\Gamma_{i}\right) \cap \operatorname{int}\left(\Gamma_{j}\right)=\varnothing$.

For the time discretisation we consider a uniform decomposition of the time interval $[0, \infty)$ into subintervals $I_{n}=\left[t_{n-1}, t_{n}\right)$ with time step $\left|I_{n}\right|=\Delta t$, such that $t_{n}=n \Delta t(n=0,1, \ldots)$.

We choose a basis $\varphi_{1}^{p}, \cdots, \varphi_{N_{s}}^{p}$ of the space $V_{h}^{p}$ of piecewise polynomial functions of degree $p$ in space (continuous and vanishing at $\partial \Gamma$ if $p \geq 1$ ) and a basis $\beta^{1, q}, \cdots, \beta^{N_{t}, q}$ of the space $V_{\Delta t}^{q}$ of piecewise polynomial functions of degree of $q$ in time (continuous and vanishing at $t=0$ if $q \geq 1$ ).

Let $\mathcal{T}_{S}=\left\{T_{1}, \cdots, T_{N_{s}}\right\}$ be the spatial mesh for $\Gamma$ and $\mathcal{T}_{T}=\left[0, t_{1}\right),\left[t_{1}, t_{2}\right), \cdots,\left[t_{N_{t}-1}, T\right)$ the time mesh for a finite subinterval $[0, T)$.

We consider the tensor product of the approximation spaces in space and time, $V_{h}^{p}$ and $V_{\Delta t}^{q}$, associated to the space-time mesh $\mathcal{T}_{S, T}=\mathcal{T}_{S} \times \mathcal{T}_{T}$, and we write

$$
V_{h, \Delta t}^{p, q}=V_{h}^{p} \otimes V_{\Delta t}^{q}
$$


In this section we discuss the projection operators onto $V_{h, \Delta t}^{p, q}$ and their approximation properties. We recall the well-known results for $V_{h}^{p}$ and $V_{\Delta t}^{q}$, which we are going to need:

Lemma 3.1. Let $\Pi_{\Delta t}$ the orthogonal projection from $L^{2}\left(\mathbb{R}_{+}\right)$to $V_{\Delta t}^{q}$ and $m \leq q$. Then for $s \in\left[-\frac{1}{2}, \frac{1}{2}\right]$

$$
\left\|f-\Pi_{\Delta t} f\right\|_{\sigma, s, \mathbb{R}_{+}} \leq C_{k} \Delta t^{q+1-s}|f|_{\sigma, q+1, \mathbb{R}_{+}} .
$$

Lemma 3.2. Let $\Pi_{h}$ the orthogonal projection from $L^{2}(\Gamma)$ to $V_{h}^{p}$ and $m \leq p$. Then for $s \in\left[-\frac{1}{2}, \frac{1}{2}\right]$ we have in the norms of $H^{s}(\Gamma)$ resp. $\widetilde{H}^{s}(\Gamma)$ :

$$
\begin{aligned}
\left\|f-\Pi_{h} f\right\|_{s, \Gamma} & \leq C h^{m+1-s}|f|_{m+1, \Gamma} \\
\| f-\left.\Pi_{h} f\right|_{s, \Gamma, *} & \leq C h^{m+1-s}|f|_{m+1, \Gamma}
\end{aligned}
$$

holds for all $f \in H^{m+1}(\Gamma) \cap \widetilde{H}^{s}(\Gamma)$.

The second estimate for $\partial \Gamma \neq \emptyset$ follows by extending $\Pi_{h} f \in V_{h}^{p}$ by zero outside $\Gamma$ which allows to estimate a $\widetilde{H}^{ \pm \frac{1}{2}}$ norm on the left hand side by standard Sobolev norms (see [14]). Combining $\Pi_{h}$ and $\Pi_{\Delta t}$ one obtains as in Proposition 3.54 of [16]:

Lemma 3.3. Let $f \in H_{\sigma}^{s}\left(\mathbb{R}^{+}, H^{m}(\Gamma) \cap \widetilde{H}^{r}(\Gamma)\right), 0<m \leq q+1,0<s \leq p+1$, $r \leq s,|l| \leq \frac{1}{2}$ such that $l r \geq 0$. Then if $l, r \leq 0$

$$
\begin{aligned}
\left\|f-\Pi_{h} \circ \Pi_{\Delta t} f\right\|_{r, l, \Gamma} & \leq C\left(h^{\alpha}+(\Delta t)^{\beta}\right)\|f\|_{s, m, \Gamma}, \\
\left\|f-\Pi_{h} \circ \Pi_{\Delta t} f\right\|_{r, l, \Gamma, *} & \leq C \log _{*}(h)\left(h^{\alpha}+(\Delta t)^{\beta}\right)\|f\|_{s, m, \Gamma},
\end{aligned}
$$

where $\alpha=\min \left\{m-l, m-\frac{m(l+r)}{m+s}\right\}, \beta=\min \left\{m+s-(l+r), m+s-\frac{m+s}{m} l\right\}$. If $l, r>0, \beta=m+s-(l+r)$.

We are also going to require inverse estimates like (3.182) in [16] for $s, m \leq 0$

$$
\left\|p_{h, \Delta t}\right\|_{0,0, \Gamma} \leq C(\Delta t)^{s} \max \left(h^{m}, \Delta t^{m}\right)\left\|p_{h, \Delta t}\right\|_{s, m, \Gamma}
$$

for $p_{h, \Delta t}$ in the approximation spaces $V_{h, \Delta t}^{p, q}$, namely

$$
\begin{gathered}
\left.\left\|p_{h, \Delta t}\right\|_{1,-\frac{1}{2}, \Gamma, *} \lesssim \frac{1}{\Delta t}\left\|p_{h, \Delta t}\right\|_{0,-\frac{1}{2}, \Gamma, *} \quad \text { (in the proof of the Theorem } 6.1\right) \\
\left\|p_{h, \Delta t}\right\|_{1,0, \Gamma} \lesssim \frac{1}{\Delta t}\left\|p_{h, \Delta t}\right\|_{0,0, \Gamma} \quad \text { (in the proof of the Theorem 6.2) } \\
\left.\left\|p_{h, \Delta t}\right\|_{0, \frac{1}{2}, \Gamma} \lesssim \frac{1}{\min \{\sqrt{\Delta t}, \sqrt{h}\}}|| p_{h, \Delta t} \|_{0,0, \Gamma} \quad \text { (in the proof of the Theorem } 6.2\right) .
\end{gathered}
$$

The above inverse inequalities hold due to the standard estimates for regular finite element functions in the usual Sobolev spaces $H^{s}(\Gamma)$ [17] on one hand, and on the other hand the weight function $e^{-\sigma t}$ does not affect these inequalities (see [1, Lemma 2]). 


\section{Frequency-domain integral operators in the ab- sorbing half-space}

We follow the approach by Bamberger and Ha-Duong [1] and first analyze an associated Helmholtz problem in the frequency domain. The analysis will be translated into results for the wave equation in the following section.

Let $\sigma>0$. For a fixed frequency $\omega$ with $\operatorname{Im} \omega>\sigma$ we consider the exterior Helmholtz problem associated to the wave equation (11) for $u^{e} \in H^{1}\left(\Omega^{e}\right)$ :

$$
\left\{\begin{array}{l}
\left(\Delta+\omega^{2}\right) u^{e}(x)=0 \quad \text { in } \Omega^{e} \\
\frac{\partial u^{e}}{\partial n}+\alpha i \omega u^{e}=\tilde{f} \quad \text { on } \Gamma \\
\frac{\partial u^{e}}{\partial n}+\alpha_{\infty} i \omega u^{e}=0 \quad \text { on } \Gamma_{\infty}
\end{array}\right.
$$

plus a Sommerfeld radiation condition at infinity. The radiation condition holds automatically since for $\operatorname{Im} \omega>\sigma$ the solution decays like $e^{-\sigma|x|}$, and hence the solution belongs to $H^{1}\left(\Omega^{e}\right)$ and not only $H_{l o c}^{1}\left(\Omega^{e}\right)$.

We also need an auxiliary interior problem for a function $u^{i} \in H^{1}\left(\Omega^{i}\right)$ :

$$
\left\{\begin{array}{l}
\left(\Delta+\omega^{2}\right) u^{i}(x)=0 \quad \text { in } \Omega^{i} \\
\frac{\partial u^{i}}{\partial n}-\alpha i \omega u^{i}=\tilde{g} \quad \text { on } \Gamma \\
\frac{\partial u^{i}}{\partial n}+\alpha_{\infty} i \omega u^{i}=0 \quad \text { on } \Gamma_{\infty}^{\prime}=\partial \mathbb{R}_{+}^{d} \backslash \Gamma_{\infty} .
\end{array}\right.
$$

The right-hand sides $\tilde{f}, \tilde{g}$ belong to $H^{-\frac{1}{2}}(\Gamma)$.

In the appendix we prove a uniqueness result:

Theorem 4.1. The problems (22) -(3) admit at most one solution for $\operatorname{Re} \alpha \geq 0$ and $\operatorname{Re} \alpha_{\infty} \geq 0$.

The next step is to explicitly construct and represent the solution of the Helmholtz equation in $\Omega^{e}$ and $\Omega^{i}$ by means of layer potentials using the representation formula.

As derived by Ochmann [18, for $d=3$ a fundamental solution to the halfspace problem is given by:

$G_{\omega}(x, y)=\frac{e^{i \omega|x-y|}}{4 \pi|x-y|}+\frac{e^{i \omega\left|x-y^{\prime}\right|}}{4 \pi\left|x-y^{\prime}\right|}+2 \beta_{\infty} e^{-\beta_{\infty}\left(x_{3}+y_{3}\right)} \int_{\infty}^{-\left(x_{3}+y_{3}\right)} e^{-\beta_{\infty} \eta} \frac{e^{i k r(\eta)}}{4 \pi r(\eta)} d \eta$,

where $r(\eta)=\sqrt{\left(x_{1}-y_{1}\right)^{2}+\left(x_{2}-y_{2}\right)^{2}+\eta^{2}}$ and $\beta_{\infty}=i \omega \alpha_{\infty}$. For $y=\left(y_{1}, y_{2}, y_{3}\right) \in$ $\mathbb{R}_{+}^{3}, y^{\prime}$ is given by $y^{\prime}=\left(y_{1}, y_{2},-y_{3}\right)$. In any dimension, $G_{\omega}$ allows to define the potential operators for the absorbing half-space as

$$
S_{\omega} p(x)=\int_{\Gamma} G_{\omega}(x, y) p(y) d s_{y}, D_{\omega} \varphi(x)=\int_{\Gamma} \frac{\partial G_{\omega}}{\partial n_{y}}(x, y) \varphi(y) d s_{y} .
$$

Using $G_{\omega}$, the solution $u$ of the Helmholtz problems admits an integral representation formula over $\Gamma$, not just $\Gamma \cup \Gamma_{\infty} \cup \Gamma_{\infty}^{\prime}$. 
Theorem 4.2. Any solution $u \in H^{1}\left(\Omega^{i}\right) \cup H^{1}\left(\Omega^{e}\right)$ of (2)-(3) satisfying the acoustic boundary conditions on $\Gamma_{\infty} \cup \Gamma_{\infty}^{\prime}$ admits a representation

$$
u=S_{\omega} p-D_{\omega} \varphi \quad \text { in } \quad \Omega^{i} \cup \Omega^{e},
$$

where

$$
\varphi=u^{i}-u^{e} \quad \text { and } \quad p=\frac{\partial u^{i}}{\partial n}-\frac{\partial u^{e}}{\partial n} \quad \text { on } \Gamma .
$$

The proof of the representation formula is standard if $\Gamma$ is replaced by $\Gamma \cup \Gamma_{\infty} \cup \Gamma_{\infty}^{\prime}$ in the definition of $S_{\omega}$ and $D_{\omega}$. The contribution from integrals over $\Gamma_{\infty}$ and $\Gamma_{\infty}^{\prime}$, however, vanishes since $G_{\omega}$ satisfies the acoustic boundary conditions.

Taking boundary values of $S_{\omega}$ and $D_{\omega}$, we obtain integral operators on $\Gamma$,

$$
\begin{array}{lrl}
V_{\omega} p(x)=2 \int_{\Gamma} G_{\omega}(x, y) p(y) d s_{y}, & K_{\omega}^{\prime} \varphi(x)=2 \int_{\Gamma} \frac{\partial}{\partial n_{x}} G_{\omega}(x, y) \varphi(y) d s_{y}, \\
K_{\omega} p(x)=2 \int_{\Gamma} \frac{\partial}{\partial n_{y}} G_{\omega}(x, y) p(y) d s_{y}, & W_{\omega} \varphi(x)=2 \int_{\Gamma} \frac{\partial^{2}}{\partial n_{x} \partial n_{y}} G_{\omega}(x, y) \varphi(y) d s_{y} .
\end{array}
$$

Here and in the following, the integrals are interpreted as distributional pairings, equivalently as principal values. As in the full space, the operators relate the traces of $u$ with $\varphi$ and $p$ :

$$
\begin{array}{lrl}
2 u^{e}=V_{\omega} p-\left(I+K_{\omega}\right) \varphi, & 2 u^{i}=V_{\omega} p+\left(I-K_{\omega}\right) \varphi \\
2 \frac{\partial u^{e}}{\partial n}=\left(-I+K_{\omega}^{\prime}\right) p-W_{\omega} \varphi, & 2 \frac{\partial u^{i}}{\partial n}=\left(I+K_{\omega}^{\prime}\right) p-W_{\omega} \varphi .
\end{array}
$$

Adding and subtracting the boundary conditions (2)-(3) on $\Gamma$, we have

$$
\left\{\begin{array}{l}
\frac{\partial u^{e}}{\partial n}+\frac{\partial u^{i}}{\partial n}-\alpha i \omega \varphi=\tilde{f}+\tilde{g}=F \\
p-\alpha i \omega\left(u^{e}+u^{i}\right)=\tilde{g}-\tilde{f}=G .
\end{array}\right.
$$

Then using the equation (4) of the trace $u$ we find the following system of integral equations:

$$
\left\{\begin{array}{l}
K_{\omega}^{\prime} p-W_{\omega} \varphi-i \omega \alpha \varphi=F \\
p-i \omega \alpha\left(V_{\omega} p-K_{\omega} \varphi\right)=G .
\end{array}\right.
$$

If $\alpha \neq 0$ multiplying the first equation by $\overline{-i \omega \psi}$ and the second by $\frac{1}{\alpha} \bar{q}$, we obtain the weak formulation after an integration by parts: Find $\tilde{\Phi}=(\varphi, p)$ such that

$$
a_{\omega}(\tilde{\Phi}, \tilde{\Psi})=l_{\omega}(\tilde{\Psi}) \quad \text { for all } \tilde{\Psi}=(\psi, q) \text {. }
$$

Here,

$$
\begin{aligned}
a_{\omega}(\tilde{\Phi}, \tilde{\Psi})=|\omega|^{2} \int_{\Gamma} \alpha \varphi \bar{\psi} d s_{x}+\int_{\Gamma} \frac{1}{\alpha} p \bar{q} d s_{x}+i \bar{\omega} \int_{\Gamma} K_{\omega}^{\prime} p \bar{\psi} d s_{x} \\
\quad-i \bar{\omega} \int_{\Gamma} W_{\omega} \varphi \bar{\psi} d s_{x}-i \omega \int_{\Gamma} V_{\omega} p \bar{q} d s_{x}+i \omega \int_{\Gamma} K_{\omega} \varphi \bar{q} d s_{x}
\end{aligned}
$$

and $l_{\omega}(\tilde{\Psi})=i \bar{\omega} \int_{\Gamma} F \bar{\psi} d s_{x}+\int_{\Gamma} \frac{1}{\alpha} G \bar{q} d s_{x}$. For $\alpha=0$, (5) reduces to $W_{\omega} \varphi=$ $K_{\omega}^{\prime} G-F$. For simplicity, we assume $\alpha^{-1}$ to exist. Other cases have to be treated differently. 
Theorem 4.3. (Coercivity)

Assume that $\operatorname{Re} \alpha>0, \operatorname{Re} \alpha_{\infty} \geq 0$. Then the following inequality holds for all $\tilde{U}=(\varphi, p) \in \widetilde{H}^{\frac{1}{2}}(\Gamma) \times L^{2}(\Gamma)$ :

$\operatorname{Re} a_{\omega}(\tilde{U}, \tilde{U}) \gtrsim \sigma\left\|\left(\operatorname{Re} \alpha^{-1}\right)^{1 / 2} p\right\|_{0, \omega, \Gamma}^{2}+\|\varphi\|_{\frac{1}{2}, \omega, \Gamma, *}^{2}+\left\|\omega(\operatorname{Re} \alpha)^{1 / 2} \varphi\right\|_{0, \omega, \Gamma}^{2}$.

Proof: Taking the real part of the bilinear form $a_{\omega}$ and using (4), we calculate

$$
\begin{aligned}
\operatorname{Re}\left(a_{\omega}(\tilde{U}, \tilde{U})\right)= & \operatorname{Re} \int_{\Gamma}\left(K_{\omega}^{\prime} p-W_{\omega} \varphi-i \omega \alpha \varphi\right)(\overline{-i \omega \varphi})+\bar{p} \frac{p-i \omega \alpha\left(V_{\omega} p-K_{\omega} \varphi\right)}{\alpha} d s_{x} \\
=\operatorname{Re} \int_{\Gamma}\left[\frac{\partial u^{i}}{\partial n}\right. & \left.+\frac{\partial u^{e}}{\partial n}-i \omega \alpha\left(u^{i}-u^{e}\right)\right] i \bar{\omega}\left(\overline{u^{i}}-\overline{u^{e}}\right) d s_{x} \\
& +\operatorname{Re} \int_{\Gamma} \frac{1}{\alpha}\left(\frac{\partial \bar{u}^{i}}{\partial n}-\frac{\partial \bar{u}^{e}}{\partial n}\right)\left(\frac{\partial u^{i}}{\partial n}-\frac{\partial u^{e}}{\partial n}-i \omega \alpha\left(u^{i}+u^{e}\right)\right) d s_{x} \\
=\operatorname{Re} \int_{\Gamma} i \bar{\omega}\left(2 \frac{\partial u^{i}}{\partial n} u^{i}-2\right. & \left.\frac{\partial u^{e}}{\partial n} \bar{u}^{e}\right) d s_{x} \\
& +\int_{\Gamma} \frac{1}{\alpha} \underbrace{\left|\frac{\partial \bar{u}^{i}}{\partial n}-\frac{\partial \bar{u}^{e}}{\partial n}\right|^{2}}_{=|p|^{2}} d s_{x}+|\omega|^{2} \int_{\Gamma} \alpha \underbrace{\left|u^{i}-u^{e}\right|^{2}}_{=|\varphi|^{2}} d s_{x} .
\end{aligned}
$$

Adding 0,

$$
-\int_{\Gamma} \frac{\partial u^{e}}{\partial n} \bar{u}^{e} d s_{x}=-\int_{\Gamma} \frac{\partial u^{e}}{\partial n} \bar{u}^{e} d s_{x}-\int_{\Gamma_{\infty}} \frac{\partial u^{e}}{\partial n} \bar{u}^{e} d s_{x}+\int_{\Gamma_{\infty}} \frac{\partial u^{e}}{\partial n} \overline{u^{e}} d s_{x}
$$

integration by parts on $\Omega^{e}$ leads to:

$$
\begin{aligned}
-\int_{\Gamma} \frac{\partial u^{e}}{\partial n} \overline{u^{e}} d s_{x} & =\int_{\Omega^{e}} \Delta u^{e} \overline{u^{e}}+\nabla u^{e} \overline{\nabla u^{e}} d x+\int_{\Gamma_{\infty}} \frac{\partial u^{e}}{\partial x_{3}} \overline{u^{e}} d s_{x} \\
& =\int_{\Omega^{e}}\left|\nabla u^{e}\right|^{2}-\omega^{2}\left|u^{e}\right|^{2} d x+\int_{\Gamma_{\infty}} \frac{\partial u^{e}}{\partial x_{3}} \overline{u^{e}} d s_{x} \\
& =\int_{\Omega^{e}}\left|\nabla u^{e}\right|^{2}-\omega^{2}\left|u^{e}\right|^{2} d x-\int_{\Gamma_{\infty}} i \alpha_{\infty} \omega\left|u^{e}\right|^{2} d s_{x} .
\end{aligned}
$$

Therefore,

$$
\begin{aligned}
-\operatorname{Re} 2 i \bar{\omega} \int_{\Gamma} \frac{\partial u^{e}}{\partial n} \overline{u^{e}} d s_{x} & =\operatorname{Re}\left(2 \int_{\Omega^{e}} i \bar{\omega}\left|\nabla u^{e}\right|^{2}-i \bar{\omega} \omega^{2}\left|u^{e}\right|^{2} d x-2 \int_{\Gamma_{\infty}}(i \bar{\omega}) i \alpha_{\infty} \omega\left|u^{e}\right|^{2} d s_{x}\right) \\
& =\operatorname{Re}\left(2 \int_{\Omega^{e}} i \bar{\omega}\left|\nabla u^{e}\right|^{2}-i \omega|\omega|^{2}\left|u^{e}\right|^{2} d x+2 \int_{\Gamma_{\infty}} \alpha_{\infty}|\omega|^{2}\left|u^{e}\right|^{2} d s_{x}\right) \\
& \geq 2 \sigma \int_{\Omega^{e}}\left|\nabla u^{e}\right|^{2}+|\omega|^{2}\left|u^{e}\right|^{2} d x+2\left(\operatorname{Re} \alpha_{\infty}\right) \int_{\Gamma_{\infty}}|\omega|^{2}\left|u^{e}\right|^{2} d s_{x}
\end{aligned}
$$

Similarly,

$$
\begin{aligned}
\operatorname{Re} 2 i \bar{\omega} \int_{\Gamma} \frac{\partial u^{i}}{\partial n} \bar{u}^{i} d s_{x} & =\operatorname{Re}\left(2 \int_{\Omega^{i}} i \bar{\omega}\left|\nabla u^{i}\right|^{2}-i \bar{\omega} \omega^{2}\left|u^{i}\right|^{2} d x+2 \int_{\Gamma_{\infty}^{\prime}} \alpha_{\infty}|\omega|^{2}\left|u^{i}\right|^{2} d s_{x}\right) \\
& \geq 2 \sigma \int_{\Omega^{i}}\left|\nabla u^{i}\right|^{2}+|\omega|^{2}\left|u^{i}\right|^{2} d x+2\left(\operatorname{Re} \alpha_{\infty}\right) \int_{\Gamma_{\infty}^{\prime}}|\omega|^{2}\left|u^{i}\right|^{2} d s_{x}
\end{aligned}
$$


We conclude

$$
\begin{aligned}
\operatorname{Re} a_{\omega}(\tilde{U}, \tilde{U}) & =\operatorname{Re} 2 i \bar{\omega} \int_{\Gamma}\left(\frac{\partial u^{i}}{\partial n} \bar{u}^{i}-\frac{\partial u^{e}}{\partial n} \overline{u^{e}}\right) d s_{x}+\int_{\Gamma} \frac{1}{\alpha}|p|^{2}+|\omega|^{2} \int_{\Gamma} \alpha|\varphi|^{2} d s_{x} \\
\geq & 2 \sigma \int_{\Omega^{i} \cup \Omega^{e}}|\nabla u|^{2}+|\omega|^{2}|u|^{2} d x+\int_{\Gamma} \operatorname{Re}\left(\frac{1}{\alpha}\right)|p|^{2} d s_{x}+|\omega|^{2} \int_{\Gamma} \operatorname{Re}(\alpha)|\varphi|^{2} d s_{x} \\
& \quad+2 \int_{\Gamma_{\infty}} \operatorname{Re}\left(\alpha_{\infty}\right)|\omega|^{2}|u|^{2} d s_{x}+2 \int_{\Gamma_{\infty}^{\prime}} \operatorname{Re}\left(\alpha_{\infty}\right)|\omega|^{2}\left|u^{i}\right|^{2} d s_{x} \\
\geq 2 \sigma \int_{\Omega^{i} \cup \Omega^{e}}|\nabla u|^{2} & +|\omega|^{2}|u|^{2} d x+\int_{\Gamma} \operatorname{Re}\left(\frac{1}{\alpha}\right)|p|^{2} d s_{x}+|\omega|^{2} \int_{\Gamma} \operatorname{Re}(\alpha)|\varphi|^{2} d s_{x} .
\end{aligned}
$$

Using the trace theorem in $\Omega^{i}$ and $\Omega^{e},\|\varphi\|_{\frac{1}{2}, \omega, \Gamma, *} \lesssim \sigma\|u\|_{1, \omega, \Omega}$, we obtain the assertion:

$$
\operatorname{Re} a_{\omega}(\tilde{U}, \tilde{U}) \gtrsim \sigma\left\|\left(\operatorname{Re} \alpha^{-1}\right)^{1 / 2} p\right\|_{0, \omega, \Gamma}^{2}+\|\varphi\|_{\frac{1}{2}, \omega, \Gamma, *}^{2}+\left\|\omega(\operatorname{Re} \alpha)^{1 / 2} \varphi\right\|_{0, \omega, \Gamma}^{2} \cdot
$$

Remark 4.4. Assume Re $\alpha_{\infty} \geq 0$. Then a similar coercivity estimate holds for the single layer potential $V_{\omega}$ :

$$
\operatorname{Re}\left\langle i \omega V_{\omega} \varphi, \varphi\right\rangle \geq C_{\sigma}\|\varphi\|_{-\frac{1}{2}, \omega, \Gamma, *}^{2} .
$$

Boundedness of the integral operators is also shown by going into $\Omega^{e} \cup \Omega^{i}$. We postpone the proof to the appendix.

Theorem 4.5. (Continuity) Assume that $\operatorname{Re} \alpha_{\infty} \geq 0$. The integral operators satisfy the following mapping properties for $p \in \widetilde{H}^{-\frac{1}{2}}(\Gamma)$ and $\varphi \in \widetilde{H}^{\frac{1}{2}}(\Gamma)$ :

$$
\begin{gathered}
\left\|V_{\omega} p\right\|_{\frac{1}{2}, \omega, \Gamma} \lesssim \sigma|\omega|\|p\|_{-\frac{1}{2}, \omega, \Gamma, *}, \\
\left\|\left.W_{\omega} \varphi\right|_{-\frac{1}{2}, \omega, \Gamma} \lesssim \sigma|\omega|\right\| \varphi \|_{\frac{1}{2}, \omega, \Gamma, *}, \\
\left\|\left(I-K_{\omega}\right) \varphi\right\|_{\frac{1}{2}, \omega, \Gamma} \lesssim_{\sigma}|\omega|\|\varphi\|_{\frac{1}{2}, \omega, \Gamma, *} \\
\left\|\left(I-K_{\omega}^{\prime}\right) p\right\|_{-\frac{1}{2}, \omega, \Gamma} \lesssim \sigma|\omega|\|p\|_{-\frac{1}{2}, \omega, \Gamma, *}
\end{gathered}
$$

The theorem translates into the boundedness of the considered bilinear form.

Theorem 4.6. Assume that $\operatorname{Re} \alpha_{\infty} \geq 0$ and $\alpha, \frac{1}{\alpha} \in L^{\infty}(\Gamma)$. The bilinear form $a_{\omega}$ is continuous on $\left(\widetilde{H}^{\frac{1}{2}}(\Gamma) \times L^{2}(\Gamma)\right) \times\left(\widetilde{H}^{\frac{1}{2}}(\Gamma) \times L^{2}(\Gamma)\right)$.

Again, we refer to the appendix for a proof.

With these results we can now state the precise weak formulation of the boundary integral equation (6) : Find $\tilde{\Phi}=(\varphi, p) \in \widetilde{H}^{\frac{1}{2}}(\Gamma) \times L^{2}(\Gamma)$ such that for all $\tilde{\Psi}=(\psi, q) \in \widetilde{H}^{\frac{1}{2}}(\Gamma) \times L^{2}(\Gamma)$ :

$$
a_{\omega}(\tilde{\Phi}, \tilde{\Psi})=l_{\omega}(\tilde{\Psi}) .
$$


Using the coercivity estimate in Theorem 4.3, we conclude the following estimate on the solution:

$$
\|p\|_{0, \omega, \Gamma}+\|\varphi\|_{\frac{1}{2}, \omega, \Gamma, *}+\|\omega \varphi\|_{0, \omega, \Gamma} \lesssim_{\sigma} \min \left\{\|\omega F\|_{-\frac{1}{2}, \omega, \Gamma},\|F\|_{0, \omega, \Gamma}\right\}+\|G\|_{0, \omega, \Gamma} .
$$

Similar results are obtained for the weak formulation of the Dirichlet problem, which reads: Find $\phi \in \widetilde{H}^{-\frac{1}{2}}(\Gamma)$ such that for all $\psi \in \widetilde{H}^{-\frac{1}{2}}(\Gamma)$ :

$$
\left\langle V_{\omega} \phi, \psi\right\rangle=\langle f, \psi\rangle \text {. }
$$

When $\operatorname{Re} \alpha_{\infty} \geq 0$, from the coercivity (7) one obtains the estimate

$$
\|\phi\|_{-\frac{1}{2}, \omega, \Gamma, *} \lesssim \sigma|\omega|\|f\|_{\frac{1}{2}, \omega, \Gamma}
$$

on the solution.

\section{Time-domain boundary integral equations for an absorbing half--space}

We consider the wave equation in $\mathbb{R}_{+}^{d}$ with acoustic boundary condition

$$
\frac{\partial u}{\partial n}-\alpha_{\infty} \frac{\partial u}{\partial t}=0 \quad \text { on } \partial \mathbb{R}_{+}^{d} .
$$

In $\mathbb{R}_{+}^{3}$ Ochmann determines the Green's function to be 8

$$
G(t-s, x, y)=\frac{\delta\left(t-s-r\left(y_{3}\right)\right)}{4 \pi r\left(y_{3}\right)}+\frac{\delta\left(t-s-r\left(-y_{3}\right)\right)}{4 \pi r\left(-y_{3}\right)}+\Sigma
$$

with

$$
\Sigma=\frac{-\alpha_{\infty}}{2 \pi} \frac{\partial}{\partial t} \frac{H\left(t-s-r\left(-y_{3}\right)\right)}{\sqrt{\left(t-s+\alpha_{\infty}\left(x_{3}+y_{3}\right)\right)^{2}+\left(\alpha_{\infty}^{2}-1\right) R^{2}}}
$$

Here $H$ denotes the Heaviside function, $R^{2}=\left(x_{1}-y_{1}\right)^{2}+\left(x_{2}-y_{2}\right)^{2}$ and $r\left( \pm y_{3}\right)^{2}=R^{2}+\left(x_{3} \mp y_{3}\right)^{2}$. The second and third terms on the right-hand side of $G$ represent the field reflected by the plane $\Gamma_{\infty}$. After a Fourier transform in $t$, one recovers from $G$ the frequency-domain Green's function $G_{\omega}$ from Section 4.

As for the Helmholtz problem the solution $u$ of the direct scattering problem (1) and its associated interior problem admits an integral representation formula over $\Gamma$, not just $\Gamma \cup \Gamma_{\infty} \cup \Gamma_{\infty}^{\prime}$. A similar representation formula in time-domain has been obtained by Becache [19] for exterior domains in $\mathbb{R}^{3}$.

Theorem 5.1. Let $u \in L^{2}\left(\mathbb{R}^{+}, H^{1}\left(\Omega^{i} \cup \Omega^{e}\right)\right) \cap H_{0}^{1}\left(\mathbb{R}^{+}, L^{2}\left(\Omega^{i} \cup \Omega^{e}\right)\right)$ be the solution of (11) for a Lipschitz boundary $\Gamma$. Then it holds in the sense of distributions $\left(x \in \Omega^{e} \cup \Omega^{i}, t \in \mathbb{R}^{+}\right)$:

$$
\begin{aligned}
u(t, x)= & \int_{\mathbb{R}^{+} \times \Gamma} \frac{\partial G}{\partial n_{y}}(t-\tau, x, y) u(\tau, y) d \tau d s_{y} \\
& -\int_{\mathbb{R}^{+} \times \Gamma} G(t-\tau, x, y) \frac{\partial u}{\partial n_{y}}(\tau, y) d \tau d s_{y},
\end{aligned}
$$


where $G$ is a fundamental solution in the half-space which satisfies the acoustic boundary conditions.

We introduce the single layer potential in time domain for a half-space with an absorbing boundary condition as

$$
S p(t, x)=\int_{\mathbb{R}^{+} \times \Gamma} G(t-\tau, x, y) p(\tau, y) d \tau d s_{y} .
$$

Specifically in 3 dimensions, this is

$$
\begin{aligned}
S p(t, x)=\frac{1}{4 \pi} & \int_{\Gamma} \frac{p(t-|x-y|, y)}{|x-y|} d s_{y}+\frac{1}{4 \pi} \int_{\Gamma} \frac{p\left(t-\left|x-y^{\prime}\right|, y\right)}{\left|x-y^{\prime}\right|} d s_{y} \\
& -\frac{\alpha_{\infty}}{2 \pi} \int_{0}^{\infty} \int_{\Gamma} \frac{\partial}{\partial s}\left[\frac{H\left(t-s-\left|x-y^{\prime}\right|\right)}{\sqrt{\left(t-s+\alpha_{\infty}\left(x_{3}+y_{3}\right)\right)^{2}+\left(\alpha_{\infty}^{2}-1\right) R^{2}}}\right] p(s, y) d s_{y} d s .
\end{aligned}
$$

The corresponding double layer potential $D$ is:

$$
D \varphi(t, x)=\int_{\mathbb{R}^{+} \times \Gamma} \frac{\partial G}{\partial n_{y}}(t-\tau, x, y) \varphi(\tau, y) d \tau d s_{y} .
$$

The function $u=S p-D \varphi$ satisfies the wave equation on $\mathbb{R}_{+}^{d} \backslash \Gamma$, and according to the representation formula

$$
\varphi=u^{i}-u^{e}, \quad p=\frac{\partial u^{i}}{\partial n}-\frac{\partial u^{e}}{\partial n} \quad \text { on } \mathbb{R}^{+} \times \Gamma .
$$

As for the Helmholtz equation we have the following trace identities:

$$
\begin{aligned}
2 u^{e} & =V p-(I+K) \varphi & 2 u^{i} & =V p+(I-K) \varphi, \\
2 \frac{\partial u^{e}}{\partial n} & =\left(-I+K^{\prime}\right) p-W \varphi & 2 \frac{\partial u^{i}}{\partial n} & =\left(I+K^{\prime}\right) p-W \varphi .
\end{aligned}
$$

The relevant boundary integral operators on $\Gamma$ are:

$$
\begin{aligned}
& V p(t, x)=2 \int_{\mathbb{R}^{+} \times \Gamma} G(t-\tau, x, y) p(\tau, y) d \tau d s_{y}, \\
& K^{\prime} \varphi(t, x)=2 \int_{\mathbb{R}^{+} \times \Gamma} \frac{\partial G}{\partial n_{x}}(t-\tau, x, y) \varphi(\tau, y) d \tau d s_{y}, \\
& K \varphi(t, x)=2 \int_{\mathbb{R}^{+} \times \Gamma} \frac{\partial G}{\partial n_{y}}(t-\tau, x, y) \varphi(\tau, y) d \tau d s_{y}, \\
& W \varphi(t, x)=2 \int_{\mathbb{R}^{+} \times \Gamma} \frac{\partial^{2} G}{\partial n_{x} \partial n_{y}}(t-\tau, x, y) \varphi(\tau, y) d \tau d s_{y} .
\end{aligned}
$$

Because $G$ is the inverse Fourier-Laplace transform $\mathcal{F}_{\omega \rightarrow t}^{-1} G_{\omega}$, see [8], these boundary integral operators are conjugates of their frequency-domain analogues: $V=\mathcal{F}_{\omega \rightarrow t}^{-1} \circ V_{\omega} \circ \mathcal{F}_{t \rightarrow \omega}$, and analogously for $K^{\prime}, K, W$.

Substituting formula (15) into the boundary condition on $\Gamma$, we obtain the following system for the unknown functions $\varphi$ and $p$

$$
\left\{\begin{array}{l}
\left(-I+K^{\prime}\right) p-W \varphi-\alpha \partial_{t}(V p-(I+K) \varphi)=2 f \\
\left(I+K^{\prime}\right) p-W \varphi+\alpha \partial_{t}(V p+(I-K) \varphi)=2 g .
\end{array}\right.
$$


Adding respectively subtracting the two equations of (16), again leads to

$$
\left\{\begin{array}{l}
K^{\prime} p-W \varphi+\alpha \frac{\partial \varphi}{\partial t}=F \\
p+\alpha\left(V \partial_{t} p-K \partial_{t} \varphi\right)=G .
\end{array}\right.
$$

Pairing these equations with test functions $\partial_{t} \psi$ respectively $\frac{q}{\alpha}$, we obtain the following space-time variational formulation:

$$
\begin{aligned}
\int_{0}^{\infty} \int_{\Gamma}\left[\left(K^{\prime} p-W \varphi\right)+\alpha \partial_{t} \varphi\right] \partial_{t} \psi d s_{x} d_{\sigma} t & =\int_{0}^{\infty} \int_{\Gamma} F \partial_{t} \psi d s_{x} d_{\sigma} t \\
\int_{0}^{\infty} \int_{\Gamma}\left[\frac{p}{\alpha}+\left(V \partial_{t} p-K \partial_{t} \varphi\right)\right] q d s_{x} d_{\sigma} t & =\int_{0}^{\infty} \int_{\Gamma} \frac{G q}{\alpha} d s_{x} d_{\sigma} t .
\end{aligned}
$$

Here $d_{\sigma} t=e^{-2 \sigma t} d t, \sigma>0$. The system can be written as

$$
a(\Phi, \Psi)=l(\Psi)
$$

where $\Phi=(\varphi, p), \Psi=(\psi, q)$ and

$$
\begin{gathered}
a(\Phi, \Psi)=\int_{0}^{\infty} \int_{\Gamma}\left(\alpha\left(\partial_{t} \varphi\right)\left(\partial_{t} \psi\right)+\frac{1}{\alpha} p q+K^{\prime} p\left(\partial_{t} \psi\right)-W \varphi\left(\partial_{t} \psi\right)+V\left(\partial_{t} p\right) q-K\left(\partial_{t} \varphi\right) q\right) d s_{x} d_{\sigma} t \\
l(\Psi)=\int_{0}^{\infty} \int_{\Gamma} F \partial_{t} \psi d s_{x} d_{\sigma} t+\int_{0}^{\infty} \int_{\Gamma} \frac{G q}{\alpha} d s_{x} d_{\sigma} t
\end{gathered}
$$

Remark 5.2. The system of equations (17) and the variational formulation (18) are the inverse Fourier-Laplace transforms of (5) and (6).

Later we will also require the time-domain mapping properties of the boundary integral operators in the energy Sobolev spaces.

Theorem 5.3. The following operators are continuous for $r \in \mathbb{R}$ :

$$
\begin{gathered}
V: H_{\sigma}^{r+1}\left(\mathbb{R}^{+}, \widetilde{H}^{-\frac{1}{2}}(\Gamma)\right) \rightarrow H_{\sigma}^{r}\left(\mathbb{R}^{+}, H^{\frac{1}{2}}(\Gamma)\right), \\
K^{\prime}: H_{\sigma}^{r+1}\left(\mathbb{R}^{+}, \widetilde{H}^{-\frac{1}{2}}(\Gamma)\right) \rightarrow H_{\sigma}^{r}\left(\mathbb{R}^{+}, H^{-\frac{1}{2}}(\Gamma)\right), \\
K: H_{\sigma}^{r+1}\left(\mathbb{R}^{+}, \widetilde{H}^{\frac{1}{2}}(\Gamma)\right) \rightarrow H_{\sigma}^{r}\left(\mathbb{R}^{+}, H^{\frac{1}{2}}(\Gamma)\right), \\
W: H_{\sigma}^{r+1}\left(\mathbb{R}^{+}, \widetilde{H}^{\frac{1}{2}}(\Gamma)\right) \rightarrow H_{\sigma}^{r}\left(\mathbb{R}^{+}, H^{-\frac{1}{2}}(\Gamma)\right) .
\end{gathered}
$$

Proof: Like the corresponding assertions in the full space [9], the theorem follows from Theorem 4.5 and Definition 2.2 by conjugation with the Fourier transform: $V=\mathcal{F}_{\omega \rightarrow t}^{-1} \circ V_{\omega} \circ \mathcal{F}_{t \rightarrow \omega}$, and analogously for $K^{\prime}, K, W$.

Together with Theorem 4.3, the mapping properties imply continuity and coercivity of the bilinear form $a(U, V)$.

Theorem 5.4. Assume that $\operatorname{Re} \alpha_{\infty} \geq 0$ and $\alpha, \frac{1}{\alpha} \in L^{\infty}(\Gamma)$. Then the bilinear form of the variational formulation (18) is continuous on $\left(H_{\sigma}^{1}\left(\mathbb{R}^{+}, \widetilde{H}^{\frac{1}{2}}(\Gamma)\right) \times H_{\sigma}^{1}\left(\mathbb{R}^{+}, L^{2}(\Gamma)\right)\right) \times$ $\left(H_{\sigma}^{1}\left(\mathbb{R}^{+}, \widetilde{H}^{\frac{1}{2}}(\Gamma)\right) \times H_{\sigma}^{1}\left(\mathbb{R}^{+}, L^{2}(\Gamma)\right)\right)$, i.e.

$$
|a(U, V)| \lesssim \sigma\left(\|p\|_{1,0, \Gamma}+\|\varphi\|_{1, \frac{1}{2}, \Gamma, *}\right)\left(\|q\|_{1,0, \Gamma}+\|\psi\|_{1, \frac{1}{2}, \Gamma, *}\right) .
$$


If $\operatorname{Re} \alpha>0$, it verifies a coercivity estimate: There exists $C_{\sigma}>0$ such that:

$$
a(U, U) \geq C_{\sigma}\left(\|p\|_{0,0, \Gamma}^{2}+\|\varphi\|_{0, \frac{1}{2}, \Gamma, *}^{2}+\left\|\partial_{t} \varphi\right\|_{0,0, \Gamma}^{2}\right) .
$$

Proof: Equations (21) and (22) follow from Theorem 4.3 and Theorem 5.3 , Concerning (22) we note that

$$
\begin{aligned}
a(U, U) & =|a(U, U)|=\left|\int_{-\infty+i \sigma}^{\infty+i \sigma} a_{\omega}(\tilde{U}, \tilde{U}) d \omega\right| \\
& \geq\left|\int_{-\infty+i \sigma}^{\infty+i \sigma} \operatorname{Re} a_{\omega}(\tilde{U}, \tilde{U}) d \omega\right| \\
& \gtrsim\|p\|_{0,0, \Gamma}^{2}+\|\varphi\|_{0, \frac{1}{2}, \Gamma, *}^{2}+\left\|\partial_{t} \varphi\right\|_{0,0, \Gamma}^{2}
\end{aligned}
$$

Similarly (21) is a consequence of (37) and Cauchy-Schwarz.

Remark 5.5. Similarly for the Dirichlet problem (see [9, (54)] and Corollary 3.50 in [16] for the full space)

$$
b(\varphi, \varphi)=\int_{0}^{\infty} \int_{\Gamma} V\left(\partial_{t} \varphi(t, x)\right) \varphi(t, x) d \sigma_{x} d_{\sigma} t \geq C_{\sigma}\|\varphi\|_{0,-\frac{1}{2}, \Gamma, *}^{2}
$$

\section{A priori estimates in the absorbing half-space}

In the following, we will restrict ourselves to a polyhedral surface $\Gamma(d=3)$ resp. a polygonal curve $(d=2)$, which satisfies the assumptions from Section 3. The error incurred by approximating a general smooth surface or curve by a polyhedral one has been studied by Nedelec [20, and by Bamberger and Ha Duong in the context of the wave equation [1].

\subsection{Dirichlet problem}

We now use the approximation results of Section 3 to discuss the convergence of Galerkin approximations to the Dirichlet problem. As in frequency domain, the mapping properties of the integral operators allow us to state the precise weak formulation of the time-dependent boundary integral equation $V \phi=f$ : Find $\phi \in H_{\sigma}^{1}\left(\mathbb{R}^{+}, \widetilde{H}^{-\frac{1}{2}}(\Gamma)\right)$ such that

$$
b(\phi, \psi)=\left\langle\partial_{t} f, \psi\right\rangle \quad \forall \psi \in H_{\sigma}^{1}\left(\mathbb{R}^{+}, \widetilde{H}^{-\frac{1}{2}}(\Gamma)\right),
$$

where

$$
\begin{aligned}
b(\phi, \psi) & =\int_{0}^{\infty} \int_{\Gamma}\left(V \partial_{t} \phi(t, x)\right) \psi(t, x) d s_{x} d_{\sigma} t \\
\left\langle\partial_{t} f, \psi\right\rangle & =\int_{0}^{\infty} \int_{\Gamma}\left(\partial_{t} f(t, x)\right) \psi(t, x) d s_{x} d_{\sigma} t
\end{aligned}
$$

Similar to the estimate (13) we obtain

$$
\|\phi\|_{r,-\frac{1}{2}, \Gamma, *} \lesssim \sigma|| f \|_{r+1, \frac{1}{2}, \Gamma}
$$


for any $r \in \mathbb{R}$, provided that Re $\alpha_{\infty} \geq 0$. In particular, a solution $\phi \in$ $H_{\sigma}^{1}\left(\mathbb{R}^{+}, \widetilde{H}^{-\frac{1}{2}}(\Gamma)\right)$ only exists provided $f \in H_{\sigma}^{2}\left(\mathbb{R}^{+}, H^{\frac{1}{2}}(\Gamma)\right)$. Coercivity assures that the solution is unique in this case.

The Galerkin formulation of (24) reads: Find $\phi_{h, \Delta t} \in V_{h, \Delta t}^{p, q}$ such that

$$
b\left(\phi_{h, \Delta t}, \psi_{h, \Delta t}\right)=\left\langle\left(\partial_{t} f\right)_{h, \Delta t}, \psi_{h, \Delta t}\right\rangle \quad \forall \psi_{h, \Delta t} \in V_{h, \Delta t}^{p, q}
$$

For the solutions of the continuous and discrete problems we obtain the following a priori error estimate:

Theorem 6.1. Assume that $\operatorname{Re} \alpha_{\infty} \geq 0$. For the solutions $\phi \in H_{\sigma}^{1}\left(\mathbb{R}^{+}, \widetilde{H}^{-\frac{1}{2}}(\Gamma)\right)$ of (24) and $\phi_{h, \Delta t} \in V_{h, \Delta t}^{p, q}$ of (26) there holds:

$$
\begin{aligned}
\left\|\phi-\phi_{h, \Delta t}\right\|_{0,-\frac{1}{2}, \Gamma, *} \lesssim\left\|\left(\partial_{t} f\right)_{h, \Delta t}-\partial_{t} f\right\|_{0, \frac{1}{2}, \Gamma} & \\
& +\inf _{\psi_{h, \Delta t}}\left\{\left(1+\frac{1}{\Delta t}\right)\left\|\phi-\psi_{h, \Delta t}\right\|_{0,-\frac{1}{2}, \Gamma, *}+\frac{1}{\Delta t}\left\|\partial_{t} \phi-\partial_{t} \psi_{h, \Delta t}\right\|_{0,-\frac{1}{2}, \Gamma, *}\right\} .
\end{aligned}
$$

If in addition $\phi \in H_{\sigma}^{s}\left(\mathbb{R}^{+}, H^{m}(\Gamma)\right)$, then

$$
\begin{aligned}
\left\|\phi-\phi_{h, \Delta t}\right\|_{0,-\frac{1}{2}, \Gamma, *} \lesssim\left\|\left(\partial_{t} f\right)_{h, \Delta t}-\partial_{t} f\right\|_{0, \frac{1}{2}, \Gamma} & \\
& +\left(\left(h^{\alpha_{1}}+\Delta t^{\beta_{1}}\right)\left(1+\frac{1}{\Delta t}\right)+\left(h^{\alpha_{2}}+\Delta t^{\beta_{2}}\right) \frac{1}{\Delta t}\right)\|\phi\|_{s, m, \Gamma},
\end{aligned}
$$

where

$$
\begin{aligned}
& \alpha_{1}=\min \left\{m+\frac{1}{2}, m-\frac{m}{2(m+s)}\right\}, \beta_{1}=\min \left\{m+s+\frac{1}{2}, m+s+\frac{m+s}{2 m}\right\}, \\
& \alpha_{2}=\min \left\{m+\frac{1}{2}, m-\frac{m}{2(m+s-1)}\right\}, \beta_{2}=\min \left\{m+s-\frac{1}{2}, m+s-1+\frac{m+s-1}{2 m}\right\}, \\
& \text { and } \quad m \geq-\frac{1}{2}, s \geq 0 .
\end{aligned}
$$

Proof: We apply the coercivity from Remark 5.5 to $\phi_{h, \Delta t}-\psi_{h, \Delta t} \in H_{\sigma}^{1}\left(\mathbb{R}^{+}, \widetilde{H}^{-\frac{1}{2}}(\Gamma)\right)$, $\psi_{h, \Delta t} \in V_{h, \Delta t}$ :

$\left\|\phi_{h, \Delta t}-\psi_{h, \Delta t}\right\|_{0,-\frac{1}{2}, \Gamma, *}^{2} \lesssim b\left(\phi_{h, \Delta t}-\phi, \phi_{h, \Delta t}-\psi_{h, \Delta t}\right)+b\left(\phi-\psi_{h, \Delta t}, \phi_{h, \Delta t}-\psi_{h, \Delta t}\right)$.

Continuity of the duality pairing is used to estimate the first term:

$$
\begin{aligned}
b\left(\phi_{h, \Delta t}-\phi, \phi_{h, \Delta t}-\psi_{h, \Delta t}\right) & =\int_{0}^{\infty} \int_{\Gamma}\left(\left(\partial_{t} f\right)_{h, \Delta t}-\partial_{t} f\right)\left(\phi_{h, \Delta t}-\psi_{h, \Delta t}\right) d s_{x} d_{\sigma} t \\
& \leq\left\|\left(\partial_{t} f\right)_{h, \Delta t}-\partial_{t} f\right\|_{0, \frac{1}{2}, \Gamma}\left\|\phi_{h, \Delta t}-\psi_{h, \Delta t}\right\|_{0,-\frac{1}{2}, \Gamma, *}
\end{aligned}
$$

The mapping properties of $V$ from Theorem 5.3 bound the second term as follows:

$$
\begin{aligned}
b\left(\phi-\psi_{h, \Delta t}, \phi_{h, \Delta t}-\psi_{h, \Delta t}\right) & \leq\left\|V \partial_{t}\left(\phi-\psi_{h, \Delta t}\right)\right\|_{-1, \frac{1}{2}, \Gamma}\left\|\phi_{h, \Delta t}-\psi_{h, \Delta t}\right\|_{1,-\frac{1}{2}, \Gamma, *} \\
& \lesssim\left\|\phi-\psi_{h, \Delta t}\right\|_{1,-\frac{1}{2}, \Gamma, *}\left\|\phi_{h, \Delta t}-\psi_{h, \Delta t}\right\|_{1,-\frac{1}{2}, \Gamma, *} .
\end{aligned}
$$


The inverse inequality in the time variable leads to

$$
b\left(\phi-\psi_{h, \Delta t}, \phi_{h, \Delta t}-\psi_{h, \Delta t}\right) \lesssim \frac{1}{\Delta t}\left\|\phi-\psi_{h, \Delta t}\right\|_{1,-\frac{1}{2}, \Gamma, *}\left\|\phi_{h, \Delta t}-\psi_{h, \Delta t}\right\|_{0,-\frac{1}{2}, \Gamma, *},
$$

so that we conclude:

$$
\left\|\phi_{h, \Delta t}-\psi_{h, \Delta t}\right\|_{0,-\frac{1}{2}, \Gamma, *} \lesssim\left\|\left(\partial_{t} f\right)_{h, \Delta t}-\partial_{t} f\right\|_{0, \frac{1}{2}, \Gamma}+\frac{1}{\Delta t}\left\|\phi-\psi_{h, \Delta t}\right\|_{1,-\frac{1}{2}, \Gamma, *} .
$$

Using the triangle inequality, one shows that

$$
\begin{aligned}
\left\|\phi-\phi_{h, \Delta t}\right\|_{0,-\frac{1}{2}, \Gamma, *} & =\left\|\phi-\psi_{h, \Delta t}\right\|_{0,-\frac{1}{2}, \Gamma, *}+\left\|\phi_{h, \Delta t}-\psi_{h, \Delta t}\right\|_{0,-\frac{1}{2}, \Gamma, *} \\
& \lesssim\left\|\left(\partial_{t} f\right)_{h, \Delta t}-\partial_{t} f\right\|_{0, \frac{1}{2}, \Gamma}+\inf _{\psi_{h, \Delta t}}\left\{\left\|\phi-\psi_{h, \Delta t}\right\|_{0,-\frac{1}{2}, \Gamma, *}+\frac{1}{\Delta t}\left\|\phi-\psi_{h, \Delta t}\right\|_{1,-\frac{1}{2}, \Gamma, *}\right\} \\
& \lesssim\left\|\left(\partial_{t} f\right)_{h, \Delta t}-\partial_{t} f\right\|_{0, \frac{1}{2}, \Gamma} \\
& \quad+\inf _{\psi_{h, \Delta t}}\left\{\left(1+\frac{1}{\Delta t}\right)\left\|\phi-\psi_{h, \Delta t}\right\|_{0,-\frac{1}{2}, \Gamma, *}+\frac{1}{\Delta t}\left\|\partial_{t} \phi-\partial_{t} \psi_{h, \Delta t}\right\|_{0,-\frac{1}{2}, \Gamma, *}\right\} .
\end{aligned}
$$

The second assertion follows from the approximation properties stated in Lemma 3.3 .

\subsection{Acoustic boundary problem}

Next, we consider the variational formulation (18) of the acoustic boundary problem:

Find $\Phi=(\varphi, p) \in H_{\sigma}^{1}\left(\mathbb{R}^{+}, \widetilde{H}^{\frac{1}{2}}(\Gamma)\right) \times H_{\sigma}^{1}\left(\mathbb{R}^{+}, L^{2}(\Gamma)\right)$ such that for all $\Psi=$ $(\psi, q) \in H_{\sigma}^{1}\left(\mathbb{R}^{+}, \widetilde{H}^{\frac{1}{2}}(\Gamma)\right) \times H_{\sigma}^{1}\left(\mathbb{R}^{+}, L^{2}(\Gamma)\right):$

$$
a(\Phi, \Psi)=l(\Psi) .
$$

We obtain an a priori estimate analogous to (12) in the frequency domain. If $\operatorname{Re} \alpha_{\infty}, \operatorname{Re} \alpha \geq 0$ and $\alpha, \frac{1}{\alpha} \in L^{\infty}(\Gamma)$, then for all

$$
\|p\|_{r, 0, \Gamma}+\|\varphi\|_{r, \frac{1}{2}, \Gamma, *}+\left\|\left.\varphi\right|_{r+1,0, \Gamma} \lesssim_{\sigma} \min \left\{\|F\|_{r+1,-\frac{1}{2}, \Gamma}, \|\left. F\right|_{r, 0, \Gamma}\right\}+\right\| G \|_{r, 0, \Gamma} .
$$

A solution in $H_{\sigma}^{1}\left(\mathbb{R}^{+}, \widetilde{H}^{\frac{1}{2}}(\Gamma)\right) \times H_{\sigma}^{1}\left(\mathbb{R}^{+}, L^{2}(\Gamma)\right)$ therefore exists provided $G \in$ $H_{\sigma}^{1}\left(\mathbb{R}^{+}, \widetilde{H}^{0}(\Gamma)\right)$ and $F \in H_{\sigma}^{2}\left(\mathbb{R}^{+}, \widetilde{H}^{-\frac{1}{2}}(\Gamma)\right)$ or $F \in H_{\sigma}^{1}\left(\mathbb{R}^{+}, \widetilde{H}^{0}(\Gamma)\right)$.

The Galerkin discretisation of (27) reads: Find $\Phi_{h, \Delta t}=\left(p_{h, \Delta t}, \varphi_{h, \Delta t}\right) \in V_{h, \Delta t}^{\tilde{p}, \tilde{q}} \times$ $V_{h, \Delta t}^{p, q}$ such that for all $\Psi_{h, \Delta t}=\left(q_{h, \Delta t}, \psi_{h, \Delta t}\right) \in V_{h, \Delta t}^{\tilde{p}, \tilde{q}} \times V_{h, \Delta t}^{p, q}$ :

$a\left(\Phi_{h, \Delta t}, \Psi_{h, \Delta t}\right)=\tilde{l}\left(\Psi_{h, \Delta t}\right):=\int_{0}^{\infty} \int_{\Gamma} F_{h, \Delta t} \partial_{t} \psi_{h, \Delta t} d s_{x} d_{\sigma} t+\int_{0}^{\infty} \int_{\Gamma} \frac{G_{h, \Delta t} q_{h, \Delta t}}{\alpha} d s_{x} d_{\sigma} t$.

We now derive an estimate for the error of the above Galerkin approximation to (18) in the norm $\||\cdot|\|_{*}$ defined by:

$$
\|\mid\| \Phi \|_{*}=\left(\|p\|_{0,0, \Gamma}^{2}+\|\varphi\|_{0, \frac{1}{2}, \Gamma, *}^{2}+\left\|\partial_{t} \varphi\right\|_{0,0, \Gamma}^{2}\right)^{\frac{1}{2}} \quad \forall \Phi=(p, \varphi) .
$$


Theorem 6.2. Assume that $\operatorname{Re} \alpha_{\infty}$, Re $\alpha \geq 0$ and $\alpha, \frac{1}{\alpha} \in L^{\infty}(\Gamma)$. For the solutions $\Phi=(p, \varphi) \in H_{\sigma}^{1}\left(\mathbb{R}^{+}, \widetilde{H}^{\frac{1}{2}}(\Gamma)\right) \times H_{\sigma}^{1}\left(\mathbb{R}^{+}, L^{2}(\Gamma)\right)$ of (18) and $\Phi_{h, \Delta t}=$ $\left(p_{h, \Delta t}, \varphi_{h, \Delta t}\right) \in V_{h, \Delta t}^{\tilde{p}, \tilde{q}} \times V_{h, \Delta t}^{p, q}$ of (29) there holds:

$$
\begin{aligned}
& \left\|p-p_{h, \Delta t}, \varphi-\varphi_{h, \Delta t}\right\|_{*} \\
& \lesssim\left\|F_{h, \Delta t}-F\right\|_{0,0, \Gamma}+\left\|G_{h, \Delta t}-G\right\|_{0,0, \Gamma} \\
& \quad+\max \left(\frac{1}{\Delta t}, \frac{1}{\sqrt{h}}\right) \inf _{\left(q_{h, \Delta t}, \psi_{h, \Delta t}\right) \in V_{h, \Delta t}^{\tilde{p}, \tilde{q}} \times V_{h, \Delta t}^{p, q}}\left(\left\|p-q_{h, \Delta t}\right\|_{1,0, \Gamma}+\left\|\varphi-\psi_{h, \Delta t}\right\|_{1, \frac{1}{2}, \Gamma}\right)
\end{aligned}
$$

If in addition $\varphi \in H_{\sigma}^{s_{1}}\left(\mathbb{R}^{+}, H^{m_{1}}(\Gamma)\right), p \in H_{\sigma}^{s_{2}}\left(\mathbb{R}^{+}, H^{m_{2}}(\Gamma)\right)$, then we have

$$
\begin{aligned}
& \|\| p-p_{h, \Delta t}, \varphi-\varphi_{h, \Delta t} \|_{*} \\
& \lesssim\left\|F_{h, \Delta t}-F\right\|_{0,0, \Gamma}+\left\|G_{h, \Delta t}-G\right\|_{0,0, \Gamma} \\
& \quad+\max \left(\frac{1}{\Delta t}, \frac{1}{\sqrt{h}}\right)\left(\left(h^{\alpha_{1}}+\Delta t^{\beta_{1}}\right)\|p\|_{s_{1}, m_{1}, \Gamma}+\left(h^{\alpha_{2}}+\Delta t^{\beta_{2}}\right)\|\varphi\|_{s_{2}, m_{2}, \Gamma}\right),
\end{aligned}
$$

where

$$
\begin{array}{cc}
\alpha_{1}=m_{1} \quad, \quad \beta_{1}=m_{1}+s_{1}-1, \\
\alpha_{2}=\min \left\{m_{2}-\frac{1}{2}, m_{2}-\frac{3 m_{2}}{2\left(m_{2}+s_{2}\right)}\right\}, & \beta_{2}=m_{2}+s_{2}-\frac{3}{2} .
\end{array}
$$

Proof: We write $\Psi=(q, \psi)$ and start with the coercivity (22) applied to $\Phi_{h, \Delta t}-\Psi_{h, \Delta t} \in H_{\sigma}^{1}\left(\mathbb{R}^{+}, \widetilde{H}^{-\frac{1}{2}}(\Gamma)\right)$ and $\Psi_{h, \Delta t} \in V_{h, \Delta t}^{\tilde{p}, \tilde{q}} \times V_{h, \Delta t}^{p, q}$ :

$$
\begin{aligned}
\left.\left\|\Phi_{h, \Delta t}-\Psi_{h, \Delta t}\right\|\right|_{*} ^{2} & \lesssim a\left(\Phi_{h, \Delta t}-\Psi_{h, \Delta t}, \Phi_{h, \Delta t}-\Psi_{h, \Delta t}\right) \\
& =a\left(\Phi_{h, \Delta t}-\Phi, \Phi_{h, \Delta t}-\Psi_{h, \Delta t}\right)+a\left(\Phi-\Psi_{h, \Delta t}, \Phi_{h, \Delta t}-\Psi_{h, \Delta t}\right) .
\end{aligned}
$$

With the help of (18), (19) and (20), the first term leads to:

$$
\begin{aligned}
& a\left(\Phi_{h, \Delta t}-\Phi, \Phi_{h, \Delta t}-\Psi_{h, \Delta t}\right)= \int_{0}^{\infty} \int_{\Gamma}\left(F_{h, \Delta t}-F\right)\left(\partial_{t} \varphi_{h, \Delta t}-\partial_{t} \psi_{h, \Delta t}\right) d s_{x} d_{\sigma} t \\
& \quad+\int_{0}^{\infty} \int_{\Gamma}\left(\frac{G_{h, \Delta t}}{\alpha}-\frac{G}{\alpha}\right)\left(p_{h, \Delta t}-q_{h, \Delta t}\right) d s_{x} d_{\sigma} t \\
& \vdots\left\|F_{h, \Delta t}-F\right\|_{0,0, \Gamma}\left\|\partial_{t} \varphi_{h, \Delta t}-\partial_{t} \psi_{h, \Delta t}\right\|_{0,0, \Gamma} \\
& \quad+\left\|G_{h, \Delta t}-G\right\|_{0,0, \Gamma}\left\|p_{h, \Delta t}-q_{h, \Delta t}\right\|_{0,0, \Gamma} \\
& \leq\left(\left\|F_{h, \Delta t}-F\right\|_{0,0, \Gamma}+\left\|G_{h, \Delta t}-G\right\|_{0,0, \Gamma}\right) \\
& \leq \quad\left(\left\|\partial_{t} \varphi_{h, \Delta t}-\partial_{t} \psi_{h, \Delta t}\right\|_{0,0, \Gamma}+\left\|p_{h, \Delta t}-q_{h, \Delta t}\right\|_{0,0, \Gamma}\right) \\
& \leq\left(\left\|F_{h, \Delta t}-F\right\|_{0,0, \Gamma}+\left\|G_{h, \Delta t}-G\right\|_{0,0, \Gamma}\right)\left\||| \Phi_{h, \Delta t}-\Psi_{h, \Delta t}\right\| \|_{*} .
\end{aligned}
$$

Due to the continuity (21) we can estimate the second term by

$$
\begin{aligned}
\left|a\left(\Phi-\Psi_{h, \Delta t}, \Phi_{h, \Delta t}-\Psi_{h, \Delta t}\right)\right| \lesssim & \left(\left\|p-q_{h, \Delta t}\right\|_{1,0, \Gamma}+\left\|\varphi-\psi_{h, \Delta t}\right\|_{1, \frac{1}{2}, \Gamma, *}\right) \\
& \cdot\left(\left\|\varphi_{h, \Delta t}-\psi_{h, \Delta t}\right\|_{1, \frac{1}{2}, \Gamma, *}+\left\|p_{h, \Delta t}-q_{h, \Delta t}\right\|_{1,0, \Gamma}\right) .
\end{aligned}
$$


Taking into account the inverse estimate from Section 3, we have

$$
\begin{aligned}
\left\|\varphi_{h, \Delta t}-\psi_{h, \Delta t}\right\|_{1, \frac{1}{2}, \Gamma, *} & \lesssim\left\|\varphi_{h, \Delta t}-\psi_{h, \Delta t}\right\|_{0, \frac{1}{2}, \Gamma, *}+\left\|\dot{\partial}_{t} \varphi_{h, \Delta t}-\partial_{t} \psi_{h, \Delta t}\right\|_{0, \frac{1}{2}, \Gamma, *} \\
& \lesssim\left\|\varphi_{h, \Delta t}-\psi_{h, \Delta t}\right\|_{0, \frac{1}{2}, \Gamma, *}+\left(h^{-\frac{1}{2}}+\Delta t^{-\frac{1}{2}}\right)\left\|\partial_{t} \varphi_{h, \Delta t}-\partial_{t} \psi_{h, \Delta t}\right\|_{0,0, \Gamma}
\end{aligned}
$$

and in time

$$
\left\|p_{h, \Delta t}-q_{h, \Delta t}\right\|_{1,0, \Gamma} \leq \frac{1}{\Delta t}\left\|p_{h, \Delta t}-q_{h, \Delta t}\right\|_{0,0, \Gamma} .
$$

Substituting (31) and (32) into (30) results in

$$
\begin{aligned}
&\left|a\left(\Phi-\Psi_{h, \Delta t}, \Phi_{h, \Delta t}-\Psi_{h, \Delta t}\right)\right| \lesssim\left(\left\|p-q_{h, \Delta t}\right\|_{1,0, \Gamma}+\left\|\varphi-\psi_{h, \Delta t}\right\|_{1, \frac{1}{2}, \Gamma, *}\right) \\
& \cdot\left(\left\|\varphi_{h, \Delta t}-\psi_{h, \Delta t}\right\|_{0, \frac{1}{2}, \Gamma, *}+\left(\frac{1}{\sqrt{h}}+\frac{1}{\sqrt{\Delta t}}\right)\left\|\partial_{t} \varphi_{h, \Delta t}-\partial_{t} \psi_{h, \Delta t}\right\|_{0,0, \Gamma}\right. \\
&\left.+\frac{1}{\Delta t}\left\|p_{h, \Delta t}-q_{h, \Delta t}\right\|_{0,0, \Gamma}\right) \\
& \lesssim \max \left(\frac{1}{\Delta t}, \frac{1}{\sqrt{h}}\right)\left(\left\|p-q_{h, \Delta t}\right\|_{1,0, \Gamma}+\left\|\varphi-\psi_{h, \Delta t}\right\|_{1, \frac{1}{2}, \Gamma}\right) \\
& .\left|\left\|\Phi_{h, \Delta t}-\Psi_{h, \Delta t} \mid\right\|_{*} .\right.
\end{aligned}
$$

Altogether, we conclude

$$
\begin{aligned}
\left\|\left|\Phi-\Phi_{h, \Delta t}\right|\right\|_{*} \lesssim & \left\|F_{h, \Delta t}-F\right\|_{0,0, \Gamma}+\left\|G_{h, \Delta t}-G\right\|_{0,0, \Gamma} \\
& +\max \left(\frac{1}{\Delta t}, \frac{1}{\sqrt{h}}\right)\left(\left\|p-q_{h, \Delta t}\right\|_{1,0, \Gamma}+\left\|\varphi-\psi_{h, \Delta t}\right\|_{1, \frac{1}{2}, \Gamma}\right) .
\end{aligned}
$$

Using the interpolation operator from Lemma 3.3. we obtain the powers of $h$ and $\Delta t$ stated in the theorem.

\section{Appendix}

Proof of Theorem 4.1: We show that interior (3) and exterior Helmholtz problems (2) with homogeneous boundary conditions $\tilde{f}=\tilde{g}=0$ admit at most one solution, $u^{i}=u^{e}=0$. To do so we multiply the Helmholtz equation (3), (2) in $\Omega^{e}$ and $\Omega^{i}$ with $i \bar{\omega} \bar{u}$ and integrate over $\Omega^{e} \cup \Omega^{i}$. We obtain

$$
\int_{\Omega^{e} \cup \Omega^{i}} \Delta u \cdot i \bar{\omega} \bar{u}+\omega^{2} u \cdot i \bar{\omega} \bar{u} d x=0 .
$$

Applying Green's first theorem to $u^{e}$ and $u^{i}$, we obtain

$$
\int_{\Omega^{e} \cup \Omega^{i}}-i \bar{\omega}|\nabla u|^{2}+i \omega|\omega|^{2}|u|^{2} d x-\int_{\Gamma} i \bar{\omega}\left(\frac{\partial u^{e}}{\partial n} \bar{u}^{e}-\frac{\partial u^{i}}{\partial n} \bar{u}^{i}\right) d s_{x}-\int_{\Gamma_{\infty} \cup \Gamma_{\infty}^{\prime}} i \bar{\omega} \frac{\partial u}{\partial n} \bar{u} d s_{x}=0 .
$$

Here, we have neglected a contribution from a large half-sphere which tends to zero as the radius of the half-sphere goes to infinity. 
We take the real part of this equality and use the boundary conditions:

$$
\begin{aligned}
& 2 \operatorname{Im} \omega \int_{\Omega^{e} \cup \Omega^{i}}|\nabla u|^{2}+|\omega|^{2}|u|^{2} d x=\operatorname{Re}\left(\int_{\Gamma}-i \bar{\omega}\left(\frac{\partial u^{e}}{\partial n} \bar{u}^{e}-\frac{\partial u^{i}}{\partial n} \bar{u}^{i}\right) d s_{x}-\int_{\Gamma_{\infty} \cup \Gamma_{\infty}^{\prime}} i \bar{\omega} \frac{\partial u}{\partial n} \bar{u} d s_{x}\right) \\
& =\operatorname{Re}\left(\int_{\Gamma}-i \bar{\omega}\left(-\alpha i \omega u^{e} \bar{u}^{e}-\alpha i \omega u^{i} \bar{u}^{i}\right) d s_{x}-i \bar{\omega} \int_{\Gamma_{\infty} \cup \Gamma_{\infty}^{\prime}}-\alpha_{\infty} i \omega u \bar{u} d s_{x}\right) \\
& =-(\operatorname{Re} \alpha) \int_{\Gamma}|\omega|^{2}\left|u^{e}\right|^{2}+|\omega|^{2}\left|u^{i}\right|^{2} d s_{x}-\left(\operatorname{Re} \alpha_{\infty}\right) \int_{\Gamma_{\infty} \cup \Gamma_{\infty}^{\prime}}|\omega|^{2}|u|^{2} d s_{x} .
\end{aligned}
$$

Since $\operatorname{Im} \omega>0$, the conditions $\operatorname{Re} \alpha \geq 0$ and $\operatorname{Re} \alpha_{\infty} \geq 0$ ensure that $u=0$ in $H^{1}\left(\Omega^{e} \cup \Omega^{i}\right)$. The uniqueness of the solution follows.

Proof of Theorem 4.5: First we prove (8).

Let be $p$ in $\widetilde{H}^{-\frac{1}{2}}(\Gamma)$ and let $v=S_{\omega} p$. Then we saw that $v$ verifies:

$$
\left\{\begin{array}{l}
\left(\Delta+\omega^{2}\right) v(x)=0 \quad \text { in } \Omega^{i} \cup \Omega^{e} \\
\frac{\partial v^{i}}{\partial n}-\frac{\partial v^{e}}{\partial n}=p \quad \text { in } \Gamma \\
v^{i}-v^{e}=0 \quad \text { in } \Gamma
\end{array}\right.
$$

Applying Green's Theorem in $\Omega^{i}$ resp. $\Omega^{e}$ we obtain

$$
-\int_{\Gamma_{\infty}^{\prime}} i \bar{\omega} \frac{\partial v^{i}}{\partial x_{3}} \bar{v}^{i} d s_{x}+\int_{\Gamma} i \bar{\omega} \frac{\partial v^{i}}{\partial n} \bar{v}^{i} d s_{x}=\int_{\Omega^{i}}-i \omega|\omega|^{2} v^{i} \bar{v}^{i} d x+\int_{\Omega^{i}} i \bar{\omega} \nabla v^{i} \nabla \bar{v}^{i} d x
$$

and

$-\int_{\Gamma_{\infty} \cup \Gamma_{\infty}^{\prime}} i \bar{\omega} \frac{\partial v}{\partial x_{3}} \bar{v} d s_{x}-\int_{\Gamma} i \bar{\omega} \frac{\partial v^{e}}{\partial n} \bar{v}^{e} d s_{x}=\int_{\Omega^{e}}-i \omega|\omega|^{2} v^{e} \overline{v^{e}} d x+\int_{\Omega^{e}} i \bar{\omega} \nabla v^{e} \nabla \overline{v^{e}} d x$

Adding the two equations (34) and (35) we get

$-\int_{\Gamma_{\infty} \cup \Gamma_{\infty}^{\prime}} i \bar{\omega} \frac{\partial v}{\partial x_{3}} \bar{v} d s_{x}+\int_{\Gamma} i \bar{\omega}\left(\frac{\partial v^{i}}{\partial n}-\frac{\partial v^{e}}{\partial n}\right) \bar{v}^{e} d s_{x}=\int_{\Omega^{e} \cup \Omega^{i}}-i \omega|\omega|^{2}|v|^{2} d x+\int_{\Omega^{e} \cup \Omega^{i}} i \bar{\omega}|\nabla v|^{2} d x$.

Using the boundary conditions on $\Gamma$ and $\partial \mathbb{R}_{+}^{3}$ we obtain

$-\int_{\Gamma_{\infty} \cup \Gamma_{\infty}^{\prime}} \alpha_{\infty}|\omega|^{2}|v|^{2} d s_{x}+\int_{\Gamma} i \bar{\omega} p \bar{v}^{e} d s_{x}=\int_{\Omega^{e} \cup \Omega^{i}}-i \omega|\omega|^{2}|v|^{2} d x+\int_{\Omega^{e} \cup \Omega^{i}} i \bar{\omega}|\nabla v|^{2} d x$.

We take the real part of this equation:

$$
-\int_{\Gamma_{\infty} \cup \Gamma_{\infty}^{\prime}} \operatorname{Re}\left(\alpha_{\infty}\right)|\omega|^{2}|v|^{2} d s_{x}+\operatorname{Re}\left(\int_{\Gamma} i \bar{\omega} p \overline{v^{e}} d s_{x}\right)=\sigma\left(\left\|v^{e}\right\|_{1, \omega, \Omega^{e}}^{2}+\left\|v^{i}\right\|_{1, \omega, \Omega^{i}}^{2}\right) .
$$

It follows from $\operatorname{Re}\left(\alpha_{\infty}\right) \geq 0$ and from the trace theorem (Lemma 1.4) that

$$
\operatorname{Re}\left(\int_{\Gamma} i \bar{\omega} p \overline{v^{e}} d s_{x}\right) \geq \sigma\left(\left\|v^{e}\right\|_{\frac{1}{2}, \omega, \Gamma}^{2}+\left\|v^{i}\right\|_{\frac{1}{2}, \omega, \Gamma}^{2}\right) .
$$


Therefore $|\omega|\|p\|_{-\frac{1}{2}, \omega, \Gamma, *}\left\|v^{e}\right\|_{\frac{1}{2}, \omega, \Gamma} \geq 2 \sigma\left\|v^{e}\right\|_{\frac{1}{2}, \omega, \Gamma}^{2}$, or $|\omega|\|p\|_{-\frac{1}{2}, \omega, \Gamma, *} \geq 2 \sigma\left\|v^{e}\right\|_{\frac{1}{2}, \omega, \Gamma}$. As $\left.v\right|_{\Gamma}=V_{\omega} p$ we obtain

$$
\left\|V_{\omega} p\right\|_{\frac{1}{2}, \omega, \Gamma} \leq \frac{|\omega|}{2 \sigma}\|p\|_{-\frac{1}{2}, \omega, \Gamma, *}
$$

We now consider the estimate (9). Let $\varphi$ in $\widetilde{H}^{\frac{1}{2}}(\Gamma)$ and let $v=-D_{\omega} \varphi$. Then we have seen that $v$ verifies:

$$
\left\{\begin{array}{l}
\left(\Delta+\omega^{2}\right) v(x)=0 \quad \text { in } \Omega^{i} \cup \Omega^{e} \\
\frac{\partial v^{i}}{\partial n}-\frac{\partial v^{e}}{\partial n}=0 \quad \text { in } \Gamma \\
v^{i}-v^{e}=\varphi \quad \text { in } \Gamma
\end{array}\right.
$$

Moreover, $\left.\frac{\partial v^{e}}{\partial n}\right|_{\Gamma}=-W_{\omega} \varphi$.

Adding (34) and (35), we obtain

$$
-\int_{\Gamma_{\infty} \cup \Gamma_{\infty}^{\prime}} i \bar{\omega} \frac{\partial v}{\partial x_{3}} \bar{v}+\int_{\Gamma} i \bar{\omega} \frac{\partial v^{e}}{\partial n}\left(\overline{v^{i}}-\overline{v^{e}}\right)=\int_{\Omega^{e} \cup \Omega^{i}}-i \omega|\omega|^{2}|v|^{2}+\int_{\Omega^{e} \cup \Omega^{i}} i \bar{\omega}|\nabla v|^{2} .
$$

Using the boundary condition on $\Gamma$ and $\Gamma_{\infty} \cup \Gamma_{\infty}^{\prime}$ leads to the following equality:

$$
-\int_{\Gamma_{\infty} \cup \Gamma_{\infty}^{\prime}} \alpha_{\infty}|\omega|^{2}|v|^{2} d s_{x}+\int_{\Gamma} i \bar{\omega} \frac{\partial v^{e}}{\partial n} \bar{\varphi} d s_{x}=\int_{\Omega^{e} \cup \Omega^{i}}-i \omega|\omega|^{2}|v|^{2} d x+\int_{\Omega^{e} \cup \Omega^{i}} i \bar{\omega}|\nabla v|^{2} d x .
$$

Its real part is given by

$$
-\left(\operatorname{Re} \alpha_{\infty}\right) \int_{\Gamma_{\infty} \cup \Gamma_{\infty}^{\prime}}|\omega|^{2}|v|^{2} d s_{x}+\operatorname{Re}\left(\int_{\Gamma} i \bar{\omega} \frac{\partial v^{e}}{\partial n} \bar{\varphi} d s_{x}\right)=\sigma\left(\left\|v^{e}\right\|_{1, \omega, \Omega^{e}}^{2}+\left\|v^{i}\right\|_{1, \omega, \Omega^{i}}^{2}\right) .
$$

As Re $\alpha_{\infty} \geq 0$ and using Cauchy-Schwarz, we conclude

$$
\left\|v^{e}\right\|_{1, \omega, \Omega^{e}}^{2} \leq \frac{1}{\operatorname{Im}(\omega)}|\omega|\|\varphi\|_{\frac{1}{2}, \omega, \Gamma, *}\left\|\frac{\partial v^{e}}{\partial n}\right\|_{-\frac{1}{2}, \omega, \Gamma} .
$$

It remains to estimate $\frac{\partial v^{e}}{\partial n}$. From Green's theorem in $\Omega^{e}$ we see that

$$
-\int_{\Gamma_{\infty}} \frac{\partial v^{e}}{\partial x_{3}} \bar{\varphi} d s_{x}-\int_{\Gamma} \frac{\partial v^{e}}{\partial n} \bar{\varphi} d s_{x}=\int_{\Omega^{e}}-\omega^{2} v^{e} \bar{\psi} d x+\int_{\Omega^{e}} \nabla v^{e} \nabla \bar{\psi} d x
$$

where $\quad \psi \in H^{1}\left(\Omega^{e}\right)$ is an extension of $\phi \in \widetilde{H}^{\frac{1}{2}}(\Gamma)$ with $\|\psi\|_{1, \omega, \Omega^{e}} \lesssim \sigma\|\phi\|_{\frac{1}{2}, \omega, \Gamma, *}$. From the trace theorem

$$
\left|\int_{\Gamma} \frac{\partial v^{e}}{\partial n} \bar{\varphi} d s_{x}\right| \leq\left\|v^{e}\right\|_{1, \omega, \Omega^{e}}\|\psi\|_{1, \omega, \Omega^{e}}
$$

we conclude

$$
\left\|\frac{\partial v^{e}}{\partial n}\right\|_{-\frac{1}{2}, \omega, \Gamma} \sup _{\left\{\phi \in \widetilde{H}^{\frac{1}{2}}(\Gamma) /\|\phi\|_{\frac{1}{2}, \omega, \Gamma, *}=1\right\}}\left|\int_{\Gamma} \frac{\partial v^{e}}{\partial n} \bar{\phi} d s_{x}\right| \leq C\left\|v^{e}\right\|_{1, \omega, \Omega^{e}} .
$$


From (36) it follows that

$$
\left\|W_{\omega} \varphi\right\|_{-\frac{1}{2}, \omega, \Gamma}=\left\|\frac{\partial v^{e}}{\partial n}\right\|_{-\frac{1}{2}, \omega, \Gamma} \leq C\left\|v^{e}\right\|_{1, \omega, \Omega^{e}} \leq C|\omega|\|\varphi\|_{\frac{1}{2}, \omega, \Gamma, *} .
$$

Using similar reasoning, we obtain the estimates (11) and (10).

Proof of Theorem 4.6; Recall that with $\tilde{U}=(\varphi, p)$ and $\tilde{V}=(\psi, q)$

$$
\begin{array}{r}
a_{\omega}(\tilde{U}, \tilde{V})=|\omega|^{2} \int_{\Gamma} \alpha \varphi \bar{\psi} d s_{x}+\int_{\Gamma} \frac{1}{\alpha} p \bar{q} d s_{x}+i \bar{\omega} \int_{\Gamma} K_{\omega}^{\prime} p \bar{\psi} d s_{x} \\
-i \bar{\omega} \int_{\Gamma} W_{\omega} \varphi \bar{\psi} d s_{x}-i \omega \int_{\Gamma} V_{\omega} p \bar{q} d s_{x}+i \omega \int_{\Gamma} K_{\omega} \varphi \bar{q} d s_{x} .
\end{array}
$$

We estimate the various terms of the bilinear form $a_{\omega}$ using Theorem 4.5

$$
\begin{aligned}
& |\omega|^{2}\left|\int_{\Gamma} \alpha \varphi \bar{\psi} d s_{x}\right| \leq C|\omega|^{2}\|\varphi\|_{0, \omega, \Gamma}\|\psi\|_{0, \omega, \Gamma} \leq C|\omega|^{2}\|\varphi\|_{\frac{1}{2}, \omega, \Gamma, *}\|\psi\|_{\frac{1}{2}, \omega, \Gamma, *}, \\
& \left|\int_{\Gamma} \frac{1}{\alpha} p \bar{q} d s_{x}\right| \leq C|| p\left\|_{0, \omega, \Gamma}\right\| q\left\|_{0, \omega, \Gamma} \leq \frac{C}{\sigma^{2}}|\omega|^{2}|| p||_{0, \omega, \Gamma}\right\| q \|_{0, \omega, \Gamma}, \\
& \left|i \bar{\omega} \int_{\Gamma} K_{\omega}^{\prime} p \bar{\psi} d s_{x}\right| \leq \frac{|\omega|}{\sigma}\left\|K_{\omega}^{\prime} p\right\|_{-\frac{1}{2}, \omega, \Gamma}\|\psi\|_{\frac{1}{2}, \omega, \Gamma, *} \\
& \leq C \frac{|\omega|^{2}}{\sigma}\|p\|_{-\frac{1}{2}, \omega, \Gamma, *}\|\psi\|_{\frac{1}{2}, \omega, \Gamma, *} \\
& \leq C \frac{|\omega|^{2}}{\sigma}\|p\|_{0, \omega, \Gamma}\|\psi\|_{\frac{1}{2}, \omega, \Gamma, *} \\
& \left|i \bar{\omega} \int_{\Gamma} W_{\omega} \varphi \bar{\psi} d s_{x}\right| \leq|\omega|\left\|W_{\omega} \varphi\right\|_{-\frac{1}{2}, \omega, \Gamma}\|\psi\|_{\frac{1}{2}, \omega, \Gamma, *} \leq C|\omega|^{2}\|\varphi\|_{\frac{1}{2}, \omega, \Gamma, *}\|\psi\|_{\frac{1}{2}, \omega, \Gamma, *}, \\
& \left|i \omega \int_{\Gamma} V_{\omega} p \bar{q} d s_{x}\right| \leq|\omega||| V_{\omega} p\left\|_{\frac{1}{2}, \omega, \Gamma}|| q\right\|_{-\frac{1}{2}, \omega, \Gamma, *} \\
& \leq C|\omega|^{2}\|p\|_{-\frac{1}{2}, \omega, \Gamma, *}\|q\|_{-\frac{1}{2}, \omega, \Gamma, *} \\
& \leq C|\omega|^{2}\|p\|_{0, \omega, \Gamma}|| q \|_{0, \omega, \Gamma} \\
& \left|i \omega \int_{\Gamma} K_{\omega} \varphi \bar{q} d s_{x}\right| \leq|\omega||| K_{\omega} \varphi\left\|_{\frac{1}{2}, \omega, \Gamma}\right\| q \|_{-\frac{1}{2}, \omega, \Gamma, *} \\
& \leq C|\omega|^{2}\|\varphi\|_{\frac{1}{2}, \omega, \Gamma, *}\|q\|_{-\frac{1}{2}, \omega, \Gamma, *} \\
& \leq C|\omega|^{2}\|\varphi\|_{\frac{1}{2}, \omega, \Gamma, *}\|q\|_{0, \omega, \Gamma}
\end{aligned}
$$

Adding the 6 inequalities we get

$$
a_{\omega}(\tilde{U}, \tilde{V}) \lesssim \sigma\left(|\omega||| p\left\|_{0, \omega, \Gamma}+|\omega|\right\| \varphi \|_{\frac{1}{2}, \omega, \Gamma, *}\right)\left(|\omega|\|q\|_{0, \omega, \Gamma}+|\omega|\|\psi\|_{\frac{1}{2}, \omega, \Gamma, *}\right) .
$$




\section{References}

[1] A. Bamberger and T. Ha Duong, Formulation variationnelle espace-temps pour le calcul par potentiel retard de la diffraction d'une onde acoustique, Math. Meth. Appl. Sci. 8 (1986), 405-435 and 598-608.

[2] I. Terrasse, Résolution mathématique et numérique des équations de Maxwell instationnaires par une méthode de potentiels retardés, $\mathrm{PhD}$ thesis, École Polytechnique, Palaiseau, 1993.

[3] T. Ha-Duong, B. Ludwig and I. Terrasse, A Galerkin BEM for transient acoustic scattering by an absorbing obstacle, Internat. J. Numer. Methods Engrg. 57 (2003), 1845-1882.

[4] A. E. Yilmaz, J.-M. Jin, E. Michielssen, Time domain adaptive integral method for surface integral equations, IEEE Trans. Antennas Propagation 52 (2004) 2692-2708.

[5] P. J. Davies, D. B. Duncan, Convolution-in-time approximations of time domain boundary integral equations, SIAM J. Sci. Comput. 35 (2013), B43B61.

[6] P. J. Davies, D. B. Duncan, Convolution spline approximations for time domain boundary integral equations, J. Integral Equations Applications, to appear (2014).

[7] F. J. Sayas, Retarded Potentials and Time Domain Boundary Integral Equations: a road-map, lecture notes, 2013.

[8] M. Ochmann, Closed form solutions for the acoustical impulse response over a masslike or an absorbing plane, J. Acoust. Soc. Am. 129 (6), 2011.

[9] T. Ha Duong, On retarded potential boundary integral equations and their discretisations, in: Topics in computational wave propagation, pp. 301-336, Lect. Notes Comput. Sci. Eng., 31, Springer, Berlin, 2003.

[10] M. Costabel, Time-dependent problems with the boundary integral equation method. In Encyclopedia of Computational Mechanics, E. Stein, R. de Borst, and J. R. Hughes, Eds. John Wiley \& Sons, Chichester, 2004,pp. 703-721.

[11] H. Gimperlein, Z. Nezhi, E. P. Stephan, A residual a posteriori error estimate for the time-domain boundary element method, in preparation.

[12] L. Banz, H. Gimperlein, Z. Nezhi, E. P. Stephan, Time domain BEM for sound radiation of tyres, in preparation.

[13] H. Triebel, Theory of Function Spaces I/II, Birkhäuser, Basel, 1983/1992.

[14] E. P. Stephan, Boundary integral equations for screen problems in $\mathbb{R}^{3}$, Integral Equations Operator Theory 10 (1987), 236 - 257. 
[15] N. Heuer, Additive Schwarz method for the p-version of the boundary element method for the single layer potential operator on a plane screen, Numer. Math. 88 (2001), 485-511.

[16] M. Glaefke, Adaptive Methods for Time Domain Boundary Integral Equations. PhD thesis, Brunel University, 2012.

[17] I. Babuska and A. K. Aziz, Survey lectures on the mathematical foundations of the finite element method. In: The Mathematical Foundations of the Finite Element Method with Applications to Partial Differential Equations, Academic Press, New York, 1972, 3-359.

[18] M. Ochmann, The complex equivalent source method for sound propagation over an impedance plane, J. Acoust. Soc. Am. 116 (6), 2004.

[19] E. Bécache, Equations intégrales pour l'équation des ondes. Cours de l'école des ondes, INRIA, 1994. Available for download at http://www-rocq.inria.fr/ becache/cours_eqinteg.ps.gz.

[20] J. C. Nedelec, Curved finite element methods for the solution of singular integral equations on surfaces in $\mathbb{R}^{3}$, Comput. Methods Appl. Mech. Engrg. 8 (1976), 61-80. 